\title{
Adjoint design sensitivity analysis and optimization of nonlinear structures using geometrical mapping approach
}

\author{
Yintao Wei $^{\mathrm{a}^{*}}$ Chonglei Zhao ${ }^{\mathrm{a}}$ Zhenhan $\mathrm{Yao}^{\mathrm{b}}$ Patrice Hauret ${ }^{\mathrm{c}}$ Xuebing $\mathrm{Li}^{\mathrm{a}}$ Michael Kaliske ${ }^{\mathrm{d}}$ \\ ${ }^{a}$ State Key Lab Automotive Safety \& Energy, Tsinghua University, Beijing, 100084, China \\ ${ }^{b}$ Department of Engineering Mechanics, Tsinghua University, Beijing, 100084, China, \\ ${ }^{\mathrm{c}}$ Mécanique Numérique - CTEU/PM/SIM/ER, Centre de Technologies Michelin, 63040 Clermont-Ferrand, \\ Cedex 9 - FRANCE \\ ${ }^{d}$ Institute for Structural Analysis, Technische Universität Dresden, Dresden, Germany
}

\begin{abstract}
A new adjoint shape design sensitivity formulation for nonlinear structures subject to contact forces is developed. The method is based on a geometrical mapping approach where shape variation is regarded as a mapping characterized by the shape variation velocity field. An adjoint variable method is developed for performing sensitivity analysis of the average shear strain in the tire belt area, which profile is properly parameterized in function of arcs, allowing explicit design velocities calculated. It can be seen that the present method is much faster than the direct differentiation method and more accurate than the classical finite difference scheme.
\end{abstract}

Keywords: shape optimization; adjoint variable method; nonlinear structures with contact forces; sensitivity analysis; geometrical mapping approach

\section{Introduction}

Sensitivity analysis in shape optimization problems is complicated by the fact that the profile of the structure may change sharply in the optimization process [1]. This issue becomes even more critical in highly nonlinear problems such as, for example, optimal design of automotive tires whose mechanical response, durability and dynamical forces/moments generated in the contact patch strongly depends on the outer profile of the structure. Shape design sensitivity analysis (DSA) is still a challenge for tire industry researchers due to the high level of nonlinearity in material, geometry and contact stresses [2]. In this case, finite element analysis obtains an optimal design at affordable computational cost.

Global optimization algorithms that combine different types of response surface approximations are more efficient than local search methods that may get trapped in local minima. The Kriging method is more robust than standard response surface fitting because it does not require assuming the form of the response surface a priori. The gradient-based Kriging (GBK) method [3] can further reduce the number of samples. However, the issue of disposing of a stable and accurate sensitivity formulation becomes even more critical in this case [3, 4]. Another way to reduce the computational cost of shape optimization is to use sample points determined by means of the Latin hypercube design minimizing pairwise correlations and maximizing inter-site distances of sample points [4, 5]. 
The material derivative approach (MDA) (see the textbook by Choi and Kim [6, 7]) has been adopted in different engineering areas by [8-20]. The adjoint variable method (AVM) [8, 21-34] allows improving computational efficiency of sensitivity evaluations. However, the theoretical derivation of AVM for shape DSA of highly nonlinear structures as tires is a challenging task in view of the complexity of kinematic relationships. Here, a new adjoint shape DSA formulation is proposed for nonlinear tire structures with contact forces. The new formulation entails two basic aspects: (i) derivation of design velocity using a geometrical mapping approach (GMA); (ii) formulation of shape DSA for nonlinear tire structures starting from the parameterized tire profile. The final goal is to perform accurate and stable DSA at low computational cost.

The idea behind GMA is that shape variations are regarded as a mapping characterized by the shape variation velocity field. Using the virtual work principle, sensitivity equations of state variables (i.e., displacements) with respect to the shape variation are formulated. The AVM for sensitivity analysis of average shear strain in the tire belt area is hence formulated. The tire profile is parameterized using parameterized arcs, which makes boundary design velocities explicitly calculable according to its design variables. A passenger radial tire is chosen as case study: remarkably, AVM is about $80 \%$ faster than direct differentiation method (DDM) in this case. This provides a useful framework for shape sensitivity analysis and optimization of highly nonlinear structures.

The paper is structured as follows. Section 2 describes design variables and objective function of the tire shape optimization problem. Section 3 presents the geometrical mapping approach that will be used for deriving sensitivity formulation. Section 4 illustrates how tire shape is parameterized and presents the definition of shape design velocity. Section 5 explains how to formulate sensitivity of displacements and displacement gradients with respect to shape variables. Sensitivity derivation for the objective function considered in this study is presented in Sections 6 and 7, respectively for the DDM and AVM. Applications to a passenger radial tire contour optimization are discussed in Section 8. Finally, Section 9 summarizes the main findings of this study.

\section{Shape Variables and Objective Function}

This article addresses nonlinear structures with contact forces, with tires being the application example. The tire shape optimization problem in this study included the five design variables shown in Figure 1: radius of the first crown arc $R_{1}$, radius of the second crown arc $R_{2}$, radius of the shoulder arc $R_{3}$, radius of the sidewall-shoulder arc $R_{4}$, meridian coordinate of the intersection point of the first and second arcs $W_{1}$, tread contact width $W_{2}$. Since $R_{4}$ is not an independent variable as its value can be known from $W_{2}$, the radius related to the shape of the inner boundary and parameters remain constant in the optimization. 


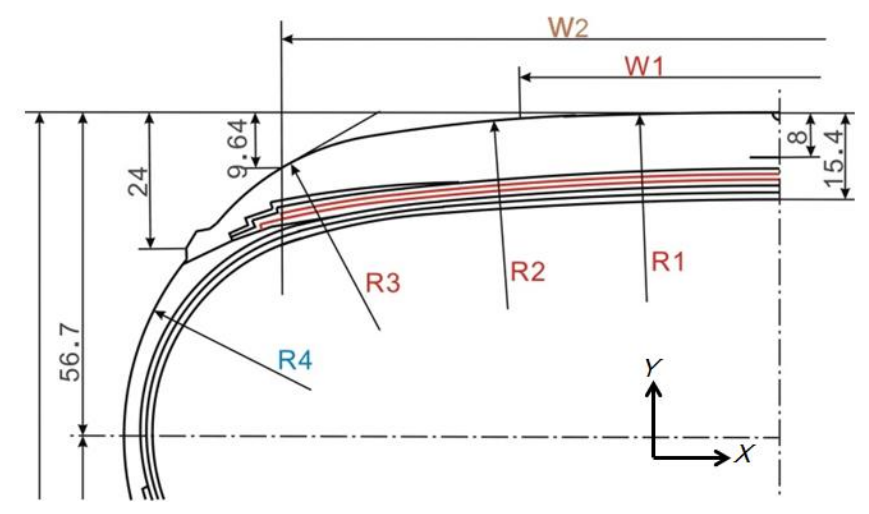

Figure 1: Design variables in the shape optimization of a tire (units/mm)

The objective function to be minimized is the average effective shear strain which develops in the belt end area near the tire-ground contact region. The corresponding area in the meridian plane is shown in Figure 2, and the 3D finite element model of tire is obtained from this 2D model by rotating around the center of tire. The circle of 360 degrees is divided into 50 sections on average and the footprint length of tire tread in the circumferential direction comes to 6 sections. Therefore the circumferential angle is taken as $2 \pi^{\prime} 6 / 50$ which is shown in Figure 4. The objective function (also called performance function) can be expressed as

$$
{ }_{\tau}^{t} \psi^{1}=\frac{1}{{ }_{\tau} V_{\mathrm{b}}} \int_{\tau}{ }_{V_{\mathrm{b}}}^{t} \bar{\varepsilon} \bar{\varepsilon}(\boldsymbol{x}) \mathrm{d}_{\tau} V
$$

Where the left subscript $\tau$ denotes the initial configuration corresponding to the shape parameter $\tau_{\alpha}(\alpha=1,2 \ldots 5)$ mentioned in Figure 1, the left superscript $t$ denotes the load parameter, ${ }_{\tau} V_{b}$ is the total volume of the design area, and ${ }_{\tau}^{t} \bar{\varepsilon}(\boldsymbol{x})$ [35] is the effective shear strain:

$$
{ }_{\tau}^{t} \bar{\varepsilon}=\sqrt{\frac{2}{3}{ }_{\tau}^{t} e_{i j}{ }_{\tau}^{t} e_{i j}}=\sqrt{\frac{2}{3}\left({ }_{\tau}^{t} \varepsilon_{i j}-\frac{1}{3}{ }_{{ }^{t}} \varepsilon_{k k} \delta_{i j}\right)\left({ }_{\tau}^{t} \varepsilon_{i j}-\frac{1}{3}{ }_{\tau}^{t} \varepsilon_{l l} \delta_{i j}\right)}
$$




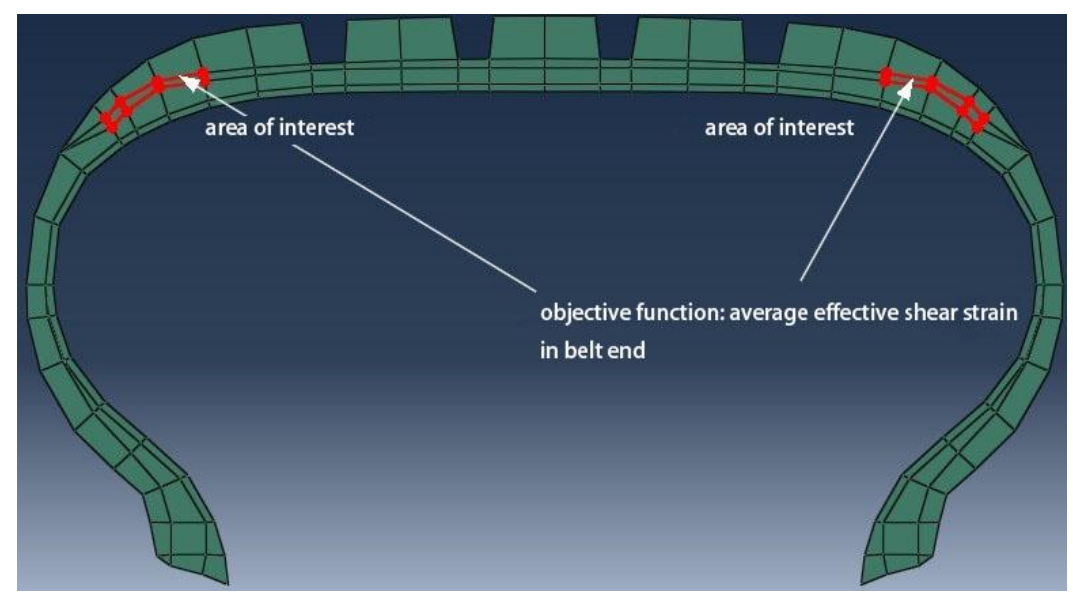

Figure 2: FE model of the meridian plane of the tire and definition of the cost function in belt end area

Figure 2 shows the FE mesh adopted for the meridian plane of the tire. The model is comprised of different rubber materials and rebar elements as reinforcements. The FE model, homogeneously divided into 50 sections in the circumferential direction, includes in most part trilinear hexahedral elements (HEX8). The level of mesh refinement was such to have mesh independent solutions at reasonably low computational cost. The structure is subject to two independent load cases: (i) inflation pressure; (ii) contact with rigid road surface under the load carried.

\section{Geometrical Mapping Approach}

In order to evaluate the sensitivity of cost function with respect to shape variations, strain sensitivities must be determined. For that purpose, sensitivity of displacements with respect to changes in shape must be obtained first. In this study, the GMA was utilized because the MDA may be misleading as the real material derivative is not suitable for formulating the variation in shape design. The partial derivative with respect to the shape parameter does not have any physical meaning, and the gradient is the derivative with respect to the Euler coordinates rather than to the Lagrange coordinates that appear in the displacement gradient of the solids. The GMA is presented with clear physical meaning, wherein the shape variation is regarded as a mapping characterized by the shape variation velocity field, or a fictitious deformation of the continuum (i.e. not a real displacement) and there are no strains or stresses corresponding to such fictitious displacement fields. The shape variation is formulated as a geometrical mapping, characterized by the shape variation velocity or shape design velocity field ${ }_{0} V_{i \alpha}$. The mapping of coordinates can be written as

$$
{ }_{\tau}^{0} x_{i}={ }_{0} x_{i}+{ }_{0} V_{i \alpha} \tau_{\alpha}={ }_{0} x_{i}+{ }_{\tau}^{0} u_{i} \quad i=1, \cdots, m ; \alpha=1, \cdots, k
$$

Where ${ }_{0} x_{i},{ }_{\tau}^{0} x_{i}$ denote the Lagrange coordinates for the original shape and the shape design corresponding to parameters $\tau_{\alpha}$ respectively, $m$ is the number of independent degrees of freedom 
(DOFs), $k$ denotes the number of design parameters, and ${ }_{\tau}^{0} u_{i}$ can be regarded as a virtual displacement, which is not a real displacement of the continuum. In addition, there are no strains or stresses corresponding to such a virtual displacement field.

For a given loading condition, the relationship between the displacements of the corresponding mapping points can be expressed as

$$
{ }_{\tau}^{t} u_{i}={ }_{0}^{t} u_{i}+{ }_{0}^{t} u_{i}^{\circ \alpha} \tau_{\alpha}
$$

Where the right superscript $o \alpha$ denotes $\partial / \partial \tau_{\alpha}$, which means the change rate of the variable for the corresponding mapping point during the shape variation, and ${ }_{0}^{t} u_{i}^{\circ \alpha}$ at $\tau_{\alpha}=0$ is the sensitivity of displacements.

The relationship between the displacement gradients of the corresponding mapping points can be expressed as

$$
{ }_{\tau}^{t} u_{i},{ }_{j}={ }_{0}^{t} u_{i},{ }_{j}+{ }_{0}^{t}\left(u_{i},{ }_{j}\right)^{\circ \alpha} \tau_{\alpha}
$$

Where ${ }_{0}^{t}\left(u_{i},{ }_{j}\right)^{\circ \alpha}$ denotes the sensitivity of displacement gradients, subscript $j$ indicates the derivative of the displacement $u_{i}$ with respect to the $j^{j^{t h}}$ coordinate, which can be formulated as:

$$
\left.{ }_{0}^{t}\left(u_{i},,_{j}\right)^{\mathrm{o} \alpha} @ \frac{\mathrm{T}}{\mathbb{\mathrm { T }} \tau_{\alpha}}\left({ }_{\tau}^{t} u_{i},,_{j}\right)\right|_{\tau=0}={ }_{0}^{t} u_{i}^{\mathrm{o} \alpha},,_{j}-{ }_{0}^{t} u_{i},{ }_{k 0} V_{k \alpha},{ }_{j}
$$

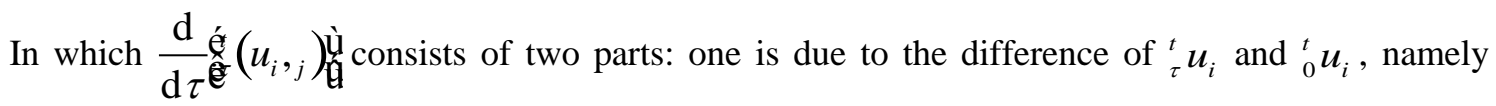
${ }_{0}^{t} u_{i}^{\circ \alpha}$; the other one comes from the difference of ${ }_{\tau}^{t}\left(u_{i},{ }_{j}\right)$ and ${ }_{0}^{t}\left(u_{i},{ }_{j}\right)$ in the case of identical ${ }_{\tau}^{t} u_{i}$ and ${ }_{0}^{t} u_{i}$

\section{Tire shape parameterization and shape design velocity calculation}

An important concept in the derivation of sensitivity is the shape design velocity. For the shape design, the boundary shape design velocity can be directly obtained from the definition used to map the coordinates (Eq. (3)):

$$
{ }_{0} V_{i \alpha}=\frac{\prod}{\llbracket \tau_{\alpha}}\left({ }_{\tau}^{0} x_{i}-{ }_{0} x_{i}\right)
$$


where the shape velocity field $V_{i \alpha}$ represents the variation relative of the $i^{\text {th }}$ degree of freedom defining the shape with respect to the $\alpha_{t h}$ design parameter (variable). Provided $\left({ }_{\tau}^{0} x_{i}-{ }_{0} x_{i}\right)$ can be formulated explicitly using the design variables $\tau_{\alpha}$. Otherwise, the finite difference approach can be followed,

$$
{ }_{0} V_{i \alpha}=\left({ }_{\tau}^{0} x_{i}\left(\Delta \tau_{\alpha}\right)-{ }_{0} x_{i}\right) / \Delta \tau_{\alpha}
$$

where $\Delta \tau_{\alpha}$ is a small increment of a design variable.

As mentioned in Section 2, we use five design variables, i.e., $R_{1}, R_{2}, R_{3}, R_{4}, W_{1}$, and $W_{2}$, as shown in Figure 1, to describe the outer profile for a series of 50-70 typical passenger car radial tires (PCR). Moreover, note that the specified data in Figure 1 for each parameter are simply an example, and $R_{4}$ can be determined by $W_{2}$. The controlling equations of all the arcs can explicitly be described as follows:

$$
\begin{aligned}
& \left(x_{1}-x_{o 1}\right)^{2}+\left(y_{1}-y_{o 1}\right)^{2}=R_{1}^{2}, \quad x_{o 1}=0, \quad y_{o 1}=D-R_{1} \\
& \left(W_{1}-x_{o 1}\right)^{2}+\left(y_{2}-y_{o 1}\right)^{2}=R_{1}^{2}, \quad\left(W_{1}-x_{o 2}\right)^{2}+\left(y_{2}-y_{o 2}\right)^{2}=R_{2}^{2} \\
& \left(W_{2}-x_{o 2}\right)^{2}+\left(\bar{y}-y_{o 2}\right)^{2}=R_{2}^{2}, \quad\left(W_{2}-x_{o 4}\right)^{2}+\left(\bar{y}-y_{o 4}\right)^{2}=R_{4}^{2}, \\
& y_{o 4}=D-H, \quad x_{o 4}=B-R_{4} \\
& \left(x_{3}-x_{o 2}\right)^{2}+\left(y_{3}-y_{o 2}\right)^{2}=R_{2}^{2}, \quad\left(x_{3}-x_{o 3}\right)^{2}+\left(y_{3}-y_{o 3}\right)^{2}=R_{3}^{2}, \\
& \left(x_{4}-x_{o 3}\right)^{2}+\left(y_{4}-y_{o 3}\right)^{2}=R_{3}^{2}, \quad\left(x_{4}-x_{o 4}\right)^{2}+\left(y_{4}-y_{o 4}\right)^{2}=R_{4}^{2} \\
& \frac{W_{1}-x_{o 2}}{y_{2}-y_{o 2}}=\frac{W_{1}-x_{o 1}}{y_{2}-y_{o 1}} ; \quad \frac{x_{3}-x_{o 2}}{y_{3}-y_{o 2}}=\frac{x_{3}-x_{o 3}}{y_{3}-y_{o 3}} ; \quad \frac{x_{4}-x_{o 3}}{y_{4}-y_{o 3}}=\frac{x_{4}-x_{o 4}}{y_{4}-y_{o 4}}
\end{aligned}
$$

where $B$ is the tire width; $D$ is the diameter; $x_{o i}, y_{o i}$ are the coordinates of the $i^{\text {th }}$ arc center; $x_{i}, y_{i}$ are outer surface point coordinates belonging to the $i^{\text {th }}$ arc.

This means that, giving the five design variables, the tire width $B$ and the diameter $D$, all 4 arcs as well as the intersection points can be calculated; therefore, the tire shape can be determined.

The design velocity calculation is a key to the shape optimization problem, which is closely related to shape parameterization. A shape change can be described by a mapping function $\boldsymbol{T}$ expressed by

$$
x_{\tau} \equiv T(x, \tau), \quad \Omega_{\tau} \equiv T(\Omega, \tau)
$$

where $\Omega$ represents the whole shape domain.

The design velocity, physically, can be understood as the partial derivative of the shape coordinates with the shape parameter. If one imagines this partial derivative as "the design velocity", the shape parameter $\tau$ can be taken as the "time", therefore the design velocity can be defined as 


$$
V\left(x_{\tau}, \tau\right) \equiv \frac{d x_{\tau}}{d \tau}=\frac{d T(x, \tau)}{d \tau}=\frac{\partial T(x, \tau)}{\partial \tau}
$$

It can be shown that the design velocity is the key parameter for mesh updating and to evaluate sensitivity of objective function with respect to shape variables.

Because all the arcs can be explicitly formulated using Eq. (9), the tire contour can be parameterized using arcs. That is:

$$
\left[\begin{array}{ll}
\mathbf{p}_{x}(u) & \mathbf{p}_{y}(u)
\end{array}\right]=\mathbf{F B}(\mathbf{R})
$$

Where $u$ are the parametric coordinates (i.e. the length of each arc); $\mathbf{F}$ and $\mathbf{B}$ are coefficient matrices, and $\mathbf{R}$ is the vector of design variables.

The real meaning of Eq. (12) is that one can explicitly calculate all outer surface point coordinates, from which the design velocity can be calculated.

Then, the design velocity of each mesh point can be determined by

$$
V=\mathbf{F} \frac{\partial \mathbf{B}}{\partial \mathbf{R}}
$$

After the $2 \mathrm{D}$ design velocity on the meridian plane is obtained, the $3 \mathrm{D}$ design velocities can be calculated by

$$
V_{x}=\bar{V}_{x}, V_{y}=\bar{V}_{y} * \cos (\theta), V_{z}=-\bar{V}_{y} * \sin (\theta)
$$

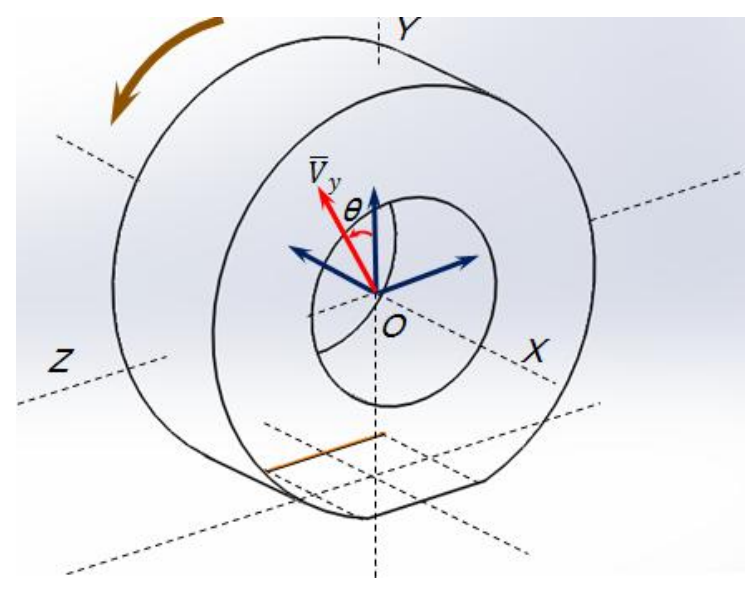

Figure 3: Sketch of the tire for 3D design velocities

The boundary design velocity can be easily calculated with Eq. (13). Various approaches have been presented in the literature [8,9], such as the boundary displacement method and the fictitious load method, for determining the domain shape design velocity field based on the boundary design velocity. The resulting domain velocity field is almost distributed across the domain, and the computation is time-consuming in general. Conversely, in the present approach, the domain velocity field is localized in one layer of finite elements, which is directly related to the design modified boundary. For HEX8 elements, the domain velocity field of the elements is directly determined by the 
boundary velocity field, and no additional computing cost is required. Furthermore, undesirable mesh distortions for composite structures can be naturally avoided.

\section{Sensitivity of displacements and displacement gradients}

The system of equations for the sensitivity of displacements can be derived from the virtual work principle. For the shape-varied structure in the shape optimization problem, the virtual work equation can be formulated as

$$
\begin{aligned}
& \grave{\mathrm{O}}_{\tau}{ }_{\tau}^{t} S_{i j} \delta_{\tau}^{t} \varepsilon_{i j} \mathrm{~d}_{\tau} V=\grave{\mathrm{O}}_{\tau} S^{\mathrm{t}}{ }_{\tau}^{t} t_{k} \delta_{0} u_{k} \mathrm{~d}_{\tau} S+\mathrm{O}_{\tau V}{ }_{\tau} \rho_{\tau}^{t} f_{k} \delta_{0} u_{k} \mathrm{~d}_{\tau} V+{ }_{\tau}^{t} C_{c}^{\mathrm{LM}} \\
& { }_{\tau}^{t} C_{c}^{\mathrm{LM}}=\grave{\mathrm{O}}_{\tau} S_{c}^{\mathrm{st}}\left({ }_{\tau}^{t} \lambda_{n} \delta_{0} u_{n}+\left({ }_{\tau}^{t} u_{n}+{ }_{\tau}^{0} g_{n}\right) \delta_{0} \lambda_{n}\right) \mathrm{d}_{\tau} S
\end{aligned}
$$

In this equation ${ }_{\tau}^{t} C_{c}^{\mathrm{LM}}$ is the contact boundary term in Lagrange multiplier approach, where $S_{\mathrm{c}}^{\text {st }}$ denotes the actually contacted part of the possibly in contact boundary, ${ }_{0} \lambda_{n},{ }_{\tau}^{t} \lambda_{n}$ denote the Lagrange multipliers while $\boldsymbol{g}_{n}$ denotes the normal gap, $\boldsymbol{u}_{i}(i=1,2,3)$ denotes the displacement, the left subscript $\tau$ denotes the initial configuration corresponding to the shape parameter $\tau_{\alpha}$, and the left superscript $t$ denotes the loading condition. The entire boundary is divided into a traction-free boundary ${ }_{\tau}^{t} S^{\mathrm{f}}$, a traction-given boundary ${ }_{\tau} S^{\mathrm{t}}$, and the contacted boundary ${ }_{\tau}^{t} S^{\text {st }}$. The traction-free boundary, including the part of the tire boundary which may come in contact with the load, is excluded from the virtual work equation. It should be noted that the division of the boundary is load and shape dependent. Here, for simplicity, frictionless contact is considered; thus, only a normal Lagrange multiplier is used.

For the shape optimization problem, the configuration corresponding to $\tau=0$ is taken as reference. Hence, Eq. (15) can be rewritten as

$$
\begin{gathered}
\grave{\mathrm{O}}_{0 V}{ }_{\tau}^{t} S_{i j} \delta_{\tau}^{t} \varepsilon_{i j}\left|{ }_{0}^{\tau} J\right| \mathrm{d}_{0} V=\text { Oे }_{0} S^{t}{ }_{\tau}^{t} t_{k} \delta_{\tau}^{t} u_{k}\left(\mathrm{~d}_{\tau} S / \mathrm{d}_{0} S\right) \mathrm{d}_{0} S+\grave{\mathrm{O}}_{0 V}{ }_{\tau} \rho_{\tau}^{t} f_{k} \delta_{\tau}^{t} u_{k}\left|{ }_{0}^{\tau} J\right| \mathrm{d}_{0} V \\
+\grave{\mathrm{O}}_{0} S_{c}\left({ }_{\tau}^{t} \lambda_{n} \delta_{0} u_{n}+\left({ }_{\tau}^{t} u_{n}+{ }_{\tau}^{0} g_{n}\right) \delta_{0} \lambda_{n}\right)\left(\mathrm{d}_{\tau} S / \mathrm{d}_{0} S\right) \mathrm{d}_{0} S
\end{gathered}
$$

Recalling Eq. (5), the strain field can be expressed as follows:

$$
\begin{aligned}
& { }_{\tau}^{t} \varepsilon_{i j}=\left({ }_{\tau}^{t} u_{i},{ }_{j}+{ }_{\tau}^{t} u_{j},{ }_{i}+{ }_{\tau}^{t} u_{k},{ }_{i}{ }_{\tau}^{t} u_{k},{ }_{j}\right) / 2=\frac{1}{2}\left\{\left({ }_{0}^{t} u_{i},{ }_{j}+{ }_{0}^{t} u_{j},{ }_{i}+{ }_{0}^{t} u_{k},{ }_{i}^{t} u_{k},{ }_{j}\right)+\right. \\
& \left.\left({ }_{0}^{t}\left(u_{i},{ }_{j}\right)^{\circ \alpha}+{ }_{0}^{t}\left(u_{j},{ }_{i}\right)^{\circ \alpha}+{ }_{0}^{t}\left(u_{k},{ }_{i}\right)^{\circ \alpha}{ }_{0}^{t} u_{k},{ }_{j}+{ }_{0}^{t} u_{k},{ }_{i}^{t}\left(u_{k},{ }_{j}\right)^{\circ \alpha}\right) \tau_{\alpha}+\left({ }_{0}^{t}\left(u_{k},{ }_{i}\right)^{\circ \alpha}{ }_{0}^{t}\left(u_{k},{ }_{j}\right)^{\circ \beta}\right) \tau_{\alpha} \tau_{\beta}\right\}
\end{aligned}
$$

where $\tau_{\alpha}$ and $\tau_{\beta}$ denote the $\alpha_{t h}$ and $\beta_{t h}$ shape design variables. Note that the strain expressed in Eq. (17) is defined according to the nonlinear theory. 
For the virtual displacement $\delta_{\tau} u_{i}$, which is arbitrary and geometrically admissible for the design shape vector $\bar{\tau}$ including all shape variables, the corresponding virtual strain can be expressed as follows:

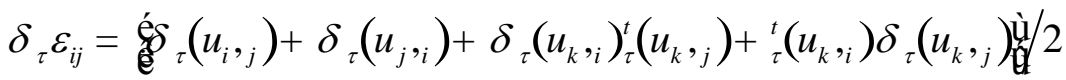

$$
\begin{aligned}
& =\frac{1}{2}\left\{\left(\delta_{0} u_{i},{ }_{j}+\delta_{0} u_{j},{ }_{i}+\delta_{0} u_{k},{ }_{i}^{t} u_{k},{ }_{j}+{ }_{0}^{t} u_{k},{ }_{i} \delta_{0} u_{k},{ }_{j}\right)+\right. \\
& +\left(\delta_{0}\left(u_{i},{ }_{j}\right)^{\circ \alpha}+\delta_{0}\left(u_{j},{ }_{i}\right)^{\circ \alpha}+\delta_{0}\left(u_{k},{ }_{i}\right)^{\circ \alpha}{ }_{0} u_{k},{ }_{j}+{ }_{0}^{t}\left(u_{k},{ }_{i}\right)^{\circ \alpha} \delta_{0} u_{k},{ }_{j}\right. \\
& \left.\left.+\delta_{0} u_{k},{ }_{i}{ }_{0}^{t}\left(u_{k},{ }_{j}\right)^{\circ \alpha}+{ }_{0}^{t} u_{k},{ }_{i} \delta_{0}\left(u_{k},{ }_{j}\right)^{\circ \alpha}\right) \tau_{\alpha}\right\}
\end{aligned}
$$

where the small higher order terms are neglected. The full derivation of Eq. (18) is given in Appendix 3.

For the second Piola-Kirchhoff stress tensor, it can be written as

$$
\begin{aligned}
& { }_{\tau}^{t} S_{i j}={ }_{0}^{t} S_{i j}+{ }_{0}^{t} S_{i j}^{\circ \alpha} \tau_{\alpha} \\
& { }_{0}^{t} S_{i j}^{\circ \alpha} \tau_{\alpha} \approx_{0}^{t} D_{\mathrm{T} i j k l}\left({ }_{0}^{t}\left(u_{k},{ }_{l}\right)^{\circ \alpha}+{ }_{0}^{t}\left(u_{l},{ }_{k}\right)^{\circ \alpha}+{ }_{0}^{t}\left(u_{m},{ }_{k}\right)^{\circ \alpha}{ }_{0}^{t} u_{m},{ }_{l}+{ }_{0}^{t} u_{m},{ }_{k}^{t}\left(u_{m},{ }_{l}\right)^{\circ \alpha}\right) \tau_{\alpha} / 2
\end{aligned}
$$

where the left term represent the stress increment, which is obtained from the product between the fourth order tangent constitutive tensor $D_{T i j k l}, i, j, k, l=1,2,3$ and the displacement gradient sensitivity tensor.

As far as it concerns the other terms in Eq. (16), it can be mentioned that

$$
{ }_{\tau} \rho={ }_{0} \rho
$$

which implies keeping the same mass density during shape changes, for a generic element $\boldsymbol{e}$ :

$$
\begin{aligned}
& \mathrm{d}_{\tau} V_{e}=\left|{ }_{0}^{\tau} \boldsymbol{J}\right| \mathrm{d}_{0} V_{e} \quad \mathrm{~d}_{\tau} V_{\mathrm{e}}=\left|{ }_{0}^{\tau} \boldsymbol{J}\right| \mathrm{d}_{0} V_{e}=\left|{ }_{0}^{\tau} \boldsymbol{J} \|_{0} \boldsymbol{J}\right| \mathrm{d} \xi_{1 \varnothing} \mathrm{d} \xi_{2 \varnothing} \mathrm{d} \xi_{3 \phi} \\
& \mathrm{d}_{\tau} V_{\mathrm{e}}=\left|{ }_{0}^{\tau} \boldsymbol{J}\right| \mathrm{d}_{0} V_{e}=\left|{ }_{0}^{\tau} \boldsymbol{J} \|_{0} \boldsymbol{J}\right| \mathrm{d} \xi_{1 \propto} \mathrm{d} \xi_{2 \varnothing} \mathrm{d} \xi_{3 \phi}
\end{aligned}
$$

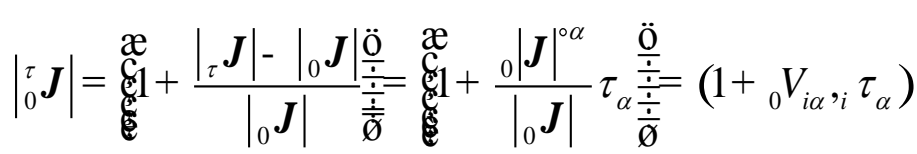

Introducing the above equations into the equation of virtual work corresponding to the shape variation - Eq. (16) - it follows: 


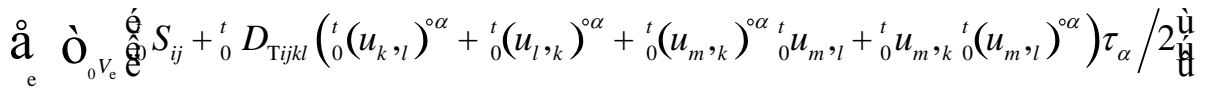

$$
\begin{aligned}
& \left.\delta_{0} u_{i},{ }_{j}+\delta_{0} u_{j}, r_{i}+\delta_{0} u_{k},{ }_{i}{ }_{0} u_{k},{ }_{j}+{ }_{0}^{t} u_{k}, \delta_{i} \delta_{0} u_{k},{ }_{j}\right) / 2+\left(\delta_{0}\left(u_{i},{ }_{j}\right)^{\circ \alpha}+\delta_{0}\left(u_{j},{ }_{i}\right)^{\circ \alpha}\right.
\end{aligned}
$$

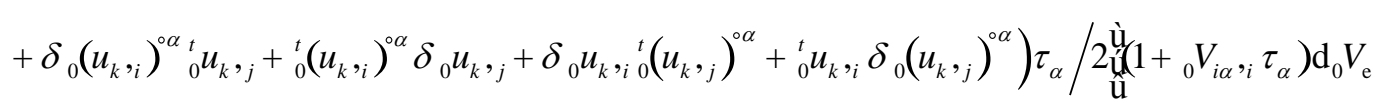

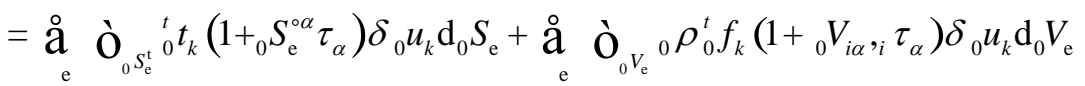

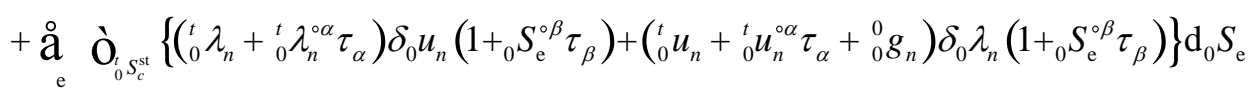

Note that $S_{\mathrm{e}}$ denotes the element surface. Developing this equation with respect to the shape variation factor $\tau_{\alpha}$, after having neglected the small higher order terms related to the stress field ${ }_{0}^{t} S_{i j}$ and the loads ${ }_{0}^{t} t_{k},{ }_{0} \rho_{0}^{t} f_{k}$, which are in equilibrium in the original shape, and introducing Eq. (6) for the sensitivity of the displacement gradient, Eq. (22) can be rewritten as 


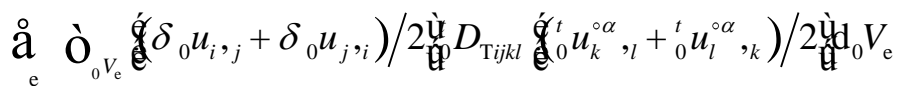

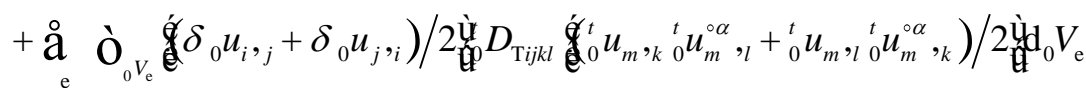

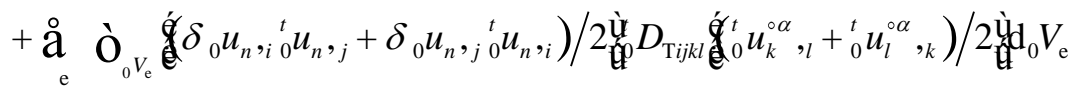

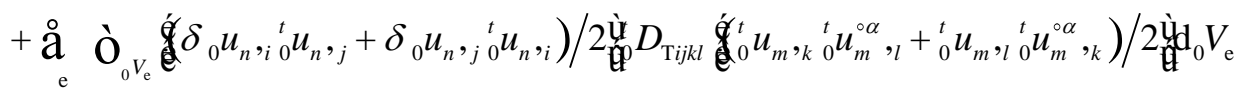

$+\stackrel{\mathrm{a}}{\mathrm{e}} \grave{\mathrm{O}}_{0}\left(V_{\mathrm{e}}\left(\delta_{0} u_{n}, r_{i}\right)_{0}^{t} S_{i j}\left({ }_{0}^{t} u_{n}^{\circ \alpha},{ }_{j}\right) \mathrm{d}_{0} V_{\mathrm{e}}\right.$

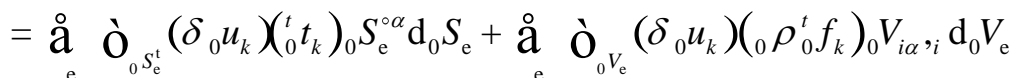

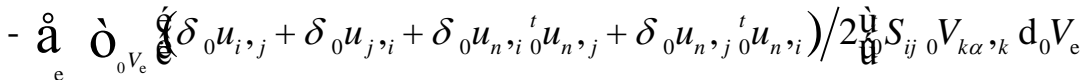

$+\underset{\mathrm{e}}{\mathrm{a}} \mathrm{O}_{0}{ }_{0} V_{\mathrm{e}}\left(\delta^{\prime}\left(\delta_{0} u_{n},{ }_{i 0}^{t} u_{n},_{k 0} V_{k \alpha},{ }_{j}+\delta_{0} u_{n},{ }_{j}{ }_{0}{ }_{n} u_{n},{ }_{0} V_{k \alpha} V_{i}\right) / 2\right)_{0}^{t} S_{i j} \grave{\mathrm{d}}_{\mathrm{d}} \mathrm{d}_{0} V_{\mathrm{e}}$

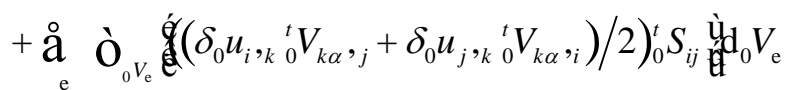

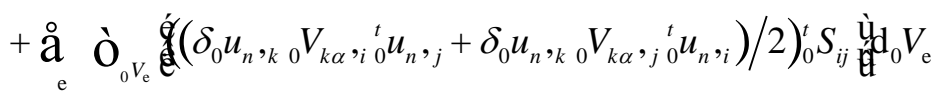

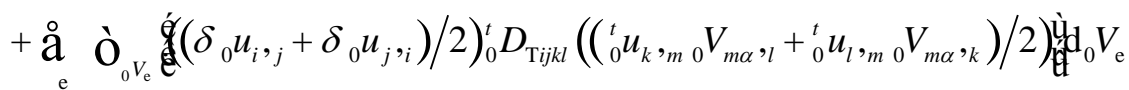

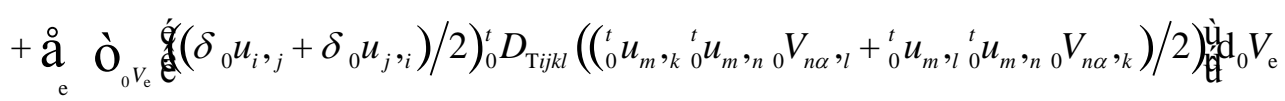

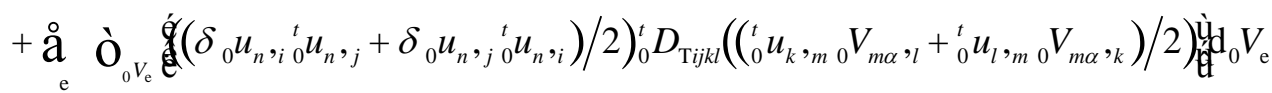

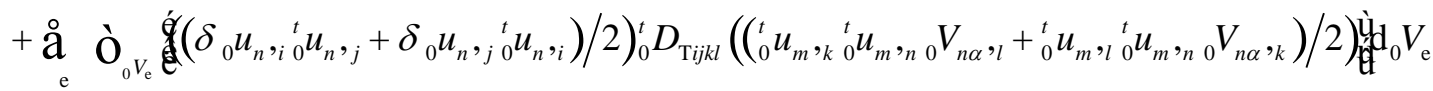

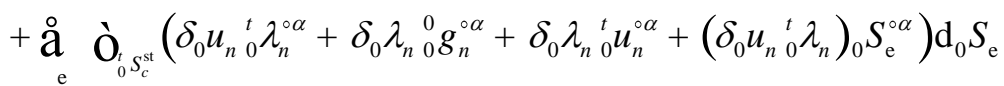

Finally, the equations for evaluating sensitivity of displacements can be formulated in matrix form as follows:

$$
\begin{aligned}
& \left({ }_{0} \boldsymbol{K}_{\mathrm{L}}+{ }_{0}^{t} \boldsymbol{K}_{\mathrm{N}}+{ }_{0}^{t} \boldsymbol{K}_{\mathrm{G}}\right){ }_{0}^{t} \boldsymbol{u}^{\circ \alpha}={ }_{0}^{t} \boldsymbol{F}^{\circ \alpha}+{ }_{0}^{t} \boldsymbol{F}_{\mathrm{C}}^{\circ \alpha}-{ }_{0}^{t} \boldsymbol{C}_{\mathrm{C} 0}^{t} \lambda^{\circ \alpha} \\
& { }_{0}^{t} \boldsymbol{G}_{\mathrm{C} 0}{ }_{0}^{t} \boldsymbol{u}^{\circ \alpha}=\boldsymbol{g}^{\circ \alpha}
\end{aligned}
$$

where $\boldsymbol{G}_{\mathrm{C}}$ is the kinematic matrix and $\boldsymbol{C}_{\mathrm{C}}$ denotes the contact matrix with regard to Lagrange multiplier, ${ }_{0} \boldsymbol{K}_{\mathrm{L}},{ }_{0}^{t} \boldsymbol{K}_{\mathrm{N}},{ }_{0}^{t} \boldsymbol{K}_{\mathrm{G}}$ are identical to those in the conventional nonlinear finite element analysis for the original design shape. That is: 


$$
\begin{aligned}
& { }_{0} \boldsymbol{K}_{\mathrm{L}}=\underset{\mathrm{e}}{\stackrel{\circ}{\mathrm{O}}} \grave{\mathrm{O}}_{0}{ }_{0} \boldsymbol{B}_{\mathrm{L}}^{\mathrm{T} t} \boldsymbol{D}_{0} \boldsymbol{B}_{\mathrm{L}} \mathrm{d}_{0} V \\
& { }_{0}^{t} \boldsymbol{K}_{\mathrm{N}}=\underset{\mathrm{e}}{\stackrel{\circ}{\mathrm{O}}}{ }_{0}\left({ }_{0} \boldsymbol{B}_{\mathrm{L}}^{\mathrm{T} t} \boldsymbol{D}_{0}^{t} \boldsymbol{B}_{\mathrm{N}}+{ }_{0}^{t} \boldsymbol{B}_{\mathrm{N} 0}^{\mathrm{T} t} \boldsymbol{D}_{0} \boldsymbol{B}_{\mathrm{L}}+{ }_{0}^{t} \boldsymbol{B}_{\mathrm{N}}^{\mathrm{T} t} \boldsymbol{D}_{0}^{t} \boldsymbol{B}_{\mathrm{N}}\right) \mathrm{d}_{0} V
\end{aligned}
$$

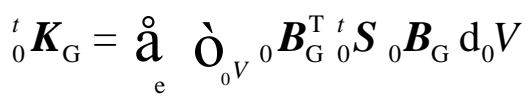

The load vector in Eq. (24) can be formulated as follows:

$$
{ }_{0}^{t} \boldsymbol{F}^{\circ \alpha}={ }_{0}^{t} \boldsymbol{Q}^{\circ \alpha}+{ }_{0}^{t} \boldsymbol{F}_{\mathrm{F}}^{\circ \alpha}
$$

Where ${ }_{0}^{t} \boldsymbol{Q}^{\circ \alpha},{ }_{0}^{t} \boldsymbol{F}_{\mathrm{F}}^{\circ \alpha}$ denote the change rates of the real loads and the fictitious loads, respectively.

For the shape-modified design, the system of equations in the nonlinear finite element analysis can be written as

$$
\left({ }_{0} \boldsymbol{K}_{\mathrm{L}}+{ }_{0} \boldsymbol{K}_{\mathrm{L}}^{\circ \alpha} \tau_{\alpha}+{ }_{0}^{t} \boldsymbol{K}_{\mathrm{N}}+{ }_{0}^{t} \boldsymbol{K}_{\mathrm{N}}^{\circ \alpha} \tau_{\alpha}+{ }_{0}^{t} \boldsymbol{K}_{\mathrm{G}}+{ }_{0}^{t} \boldsymbol{K}_{\mathrm{G}}^{\circ \alpha} \tau_{\alpha}\right)\left({ }_{0}^{t} \boldsymbol{u}+{ }_{0}^{t} \boldsymbol{u}^{\circ \alpha} \tau_{\alpha}\right)={ }_{0}^{t} \boldsymbol{F}+{ }_{0}^{t} \boldsymbol{F}_{\mathrm{R}}^{\circ \alpha} \tau_{\alpha}
$$

In the sensitivity analysis, the system of equations to be solved is

$$
\left({ }_{0} \boldsymbol{K}_{\mathrm{L}}+{ }_{0}^{t} \boldsymbol{K}_{\mathrm{N}}+{ }_{0}^{t} \boldsymbol{K}_{\mathrm{G}}\right)_{0}^{t} \boldsymbol{u}^{\circ \alpha}={ }_{0}^{t} \boldsymbol{F}_{\mathrm{R}}^{\circ \alpha}-\left({ }_{0} \boldsymbol{K}_{\mathrm{L}}^{\circ \alpha}+{ }_{0}^{t} \boldsymbol{K}_{\mathrm{N}}^{\circ \alpha}+{ }_{0}^{t} \boldsymbol{K}_{\mathrm{G}}^{\circ \alpha}\right)_{0}^{t} \boldsymbol{u}={ }_{0}^{t} \boldsymbol{F}_{\mathrm{R}}^{\circ \alpha}+{ }_{0}^{t} \boldsymbol{F}_{\mathrm{F}}^{\circ \alpha}={ }_{0}^{t} \boldsymbol{F}^{\circ \alpha}
$$

where the fictitious load ${ }_{0}^{t} \boldsymbol{F}_{\mathrm{F}}^{\circ \alpha}$ can be formulated as

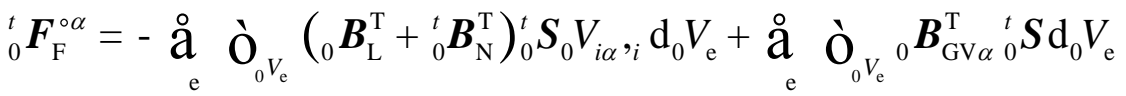

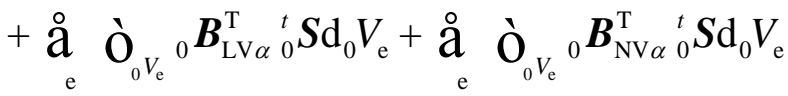

$$
\begin{aligned}
& +\underset{\mathrm{e}}{\mathrm{a}} \mathrm{O}_{0}{ }_{0}{ }_{\mathrm{c}} \boldsymbol{B}_{\mathrm{L}}^{\mathrm{T} t} \boldsymbol{D} \boldsymbol{D} \boldsymbol{E}_{\mathrm{LV} \alpha} \mathrm{d}_{0} V_{\mathrm{e}}+\underset{\mathrm{e}}{\stackrel{\circ}{\mathrm{O}}} \grave{\mathrm{O}}_{0}{ }_{0}{ }_{0} \boldsymbol{B}_{\mathrm{L}}^{\mathrm{T} t}{ }_{0} \boldsymbol{D} \boldsymbol{E}_{\mathrm{NV} \alpha} \mathrm{d}_{0} V_{\mathrm{e}}
\end{aligned}
$$

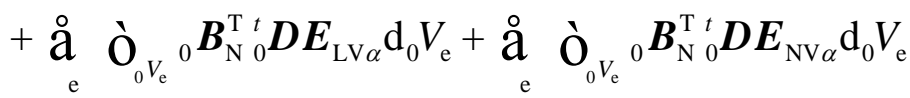

where all the terms can be derived from Eq. (23). In Eqs. (25-27), stiffness matrix ${ }_{0}^{t} \boldsymbol{K}_{\mathrm{N}},{ }_{0}^{t} \boldsymbol{K}_{\mathrm{N}},{ }_{0}^{t} \boldsymbol{K}_{\mathrm{N}}$ including strain matrix $\boldsymbol{B}_{\mathrm{L}}, \boldsymbol{B}_{\mathrm{N}}, \boldsymbol{B}_{\mathrm{G}}$ preserve their classical meaning. However, the strain matrix parameters $\boldsymbol{B}_{\mathrm{LV} \alpha}, \boldsymbol{B}_{\mathrm{NV} \alpha}, \boldsymbol{E}_{\mathrm{LV} \alpha}, \boldsymbol{E}_{\mathrm{NV} \alpha}$ related to design velocity in Eq. (31) are different from those defined in conventional finite element analysis as explained in Appendix 4. The complete derivation is a routine process and omitted here for the sake of brevity.

On the other hand, the change rates of the real loads can be calculated as 
${ }_{0}^{t} \boldsymbol{F}_{\mathrm{R}}^{\circ \alpha \mathrm{o}}{ }_{0}^{t} \boldsymbol{Q}^{\circ \alpha}=\underset{\mathrm{e}}{\mathrm{a}} \grave{\mathrm{O}}_{0} V_{\mathrm{e}} \boldsymbol{N}^{\mathrm{T}}\left({ }_{0} \rho_{0}^{t} \boldsymbol{f}\right){ }_{0} V_{i \alpha},_{i} \mathrm{~d}_{0} V_{\mathrm{e}}+\underset{\mathrm{e}}{\stackrel{\mathrm{a}}{\mathrm{O}_{0} S_{\mathrm{e}}}} \boldsymbol{N}^{\mathrm{T}}{ }_{0}^{t} \boldsymbol{t}_{0} S_{\mathrm{e}}^{\circ \alpha} \mathrm{d}_{0} S_{\mathrm{e}}$

Sensitivity of displacements can be determined from the linear system(30).

\section{Determination of Cost Function Sensitivities with the Direct Differentiation Method}

The sensitivities of cost function defined by Eq. (1) can be computed once sensitivities of displacements and the displacement gradients are known. The average strain functional (1) defined for the tire shape optimization problem can be expressed as

$$
{ }_{0}^{t} \psi=\int_{0} f\left({ }_{0}^{t} u_{i}\left({ }_{0} \boldsymbol{x}\right),{ }_{0}^{t} u_{i},{ }_{j}\left({ }_{0} \boldsymbol{x}\right)\right) \mathrm{d}_{0} V
$$

If we assume that density remains unchanged during the shape variation, the cost function can be formulated as

$$
\begin{aligned}
{ }_{\tau}^{t} \psi & =\int_{\tau V} f\left({ }_{\tau}^{t} u_{i}\left({ }_{\tau} \boldsymbol{x}\right),{ }_{\tau}^{t} u_{i},{ }_{j}\left({ }_{\tau} \boldsymbol{x}\right)\right) \mathrm{d}_{\tau} V \\
& =\int_{0} f\left({ }_{0}^{t} u_{i}\left({ }_{0} \boldsymbol{x}\right)+{ }_{0}^{t} u_{i}^{\mathrm{o} \alpha}\left({ }_{0} \boldsymbol{x}\right) \tau_{\alpha},{ }_{0}^{t} u_{i},{ }_{j}\left({ }_{0} \boldsymbol{x}\right)+{ }_{0}^{t}\left(u_{i},{ }_{j}\right){ }^{\mathrm{o} \alpha} \tau_{\alpha}\right)\left[1+\left({ }_{0}|\boldsymbol{J}|^{\mathrm{o} \beta} /\left.\right|_{0} \boldsymbol{J} \mid\right) \tau_{\beta}\right] \mathrm{d}_{0} V \\
& \approx_{0}^{t} \psi+{ }_{0}^{t} \psi^{\mathrm{o} \alpha} \tau_{\alpha}
\end{aligned}
$$

where

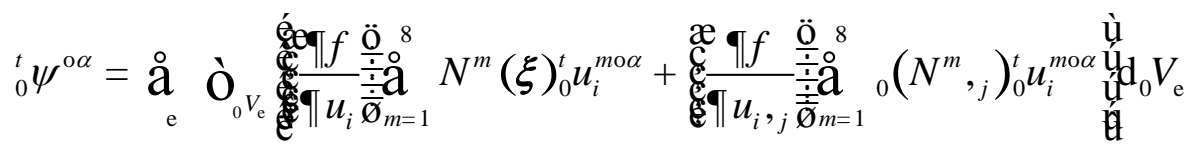

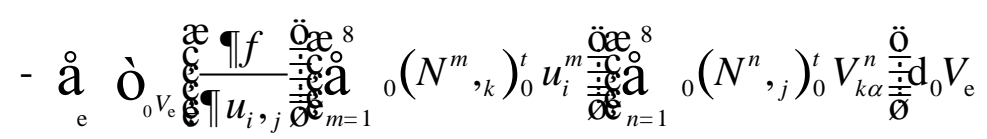

$$
\begin{aligned}
& +\underset{\mathrm{e}}{\mathrm{Q}} \mathrm{O}_{0_{0} V_{\mathrm{e}}} f\left({ }_{0}^{t} u_{i}\left({ }_{0} x\right),{ }_{0}^{t} u_{i},{ }_{j}\left({ }_{0} x\right)\right) \stackrel{\mathrm{a}}{m=1}_{0}^{8}\left(N^{m},{ }_{i}\right)_{0} V_{i \alpha}^{m} \mathrm{~d}_{0} V_{\mathrm{e}}
\end{aligned}
$$

Introducing

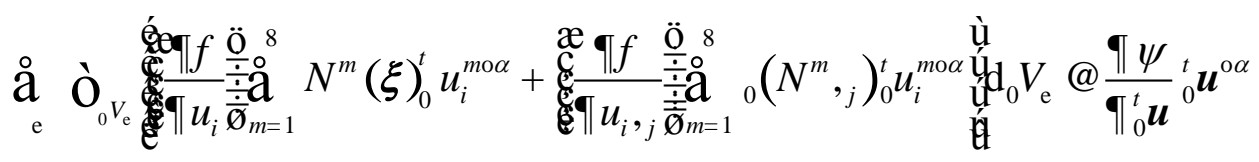

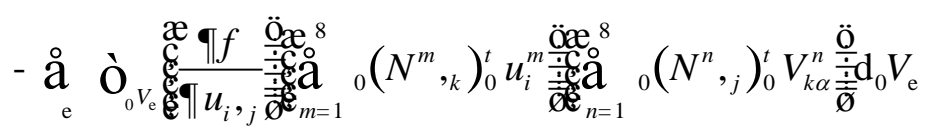

$$
\begin{aligned}
& +\underset{\mathrm{e}}{\stackrel{\circ}{\mathrm{O}_{0} V_{\mathrm{e}}}} f\left({ }_{0}^{t} u_{i}\left({ }_{0} \boldsymbol{x}\right),{ }_{0}^{t} u_{i},{ }_{j}\left({ }_{0} \boldsymbol{x}\right)\right) \mathrm{a}_{m=1}^{8}{ }_{0}\left(N^{m},_{i}\right)_{0} V_{i \alpha}^{m} \mathrm{~d}_{0} V_{\mathrm{e}} @_{0}^{t} \bar{\psi}^{\mathrm{o} \alpha}
\end{aligned}
$$


The sensitivity of cost function can hence be expressed as

$$
{ }_{0}^{t} \psi^{\mathrm{o} \alpha}=\frac{\mathbb{\coprod}_{0}^{t} \psi}{\boldsymbol{\prod}_{0}^{t} \boldsymbol{u}}{ }_{0}^{t} \boldsymbol{u}^{\mathrm{o} \alpha}+{ }_{0}^{t} \bar{\psi}^{\mathrm{o} \alpha}
$$

Displacement sensitivities ${ }_{0}^{t} \boldsymbol{u}^{\mathrm{o} \alpha}$ are obtained by solving the Eq. (30). Determination of cost function sensitivities with Eq. (38) and (30) is called direct sensitivity calculation (DDM). Since this method is computationally expensive as it requires solving the system (30), the AVM was developed in this study.

\section{Determination of Cost Function Sensitivities with the Adjoint Variable Method}

The system (24) that allows sensitivity of displacements with respect to shape variables to be computed can be rewritten as follows

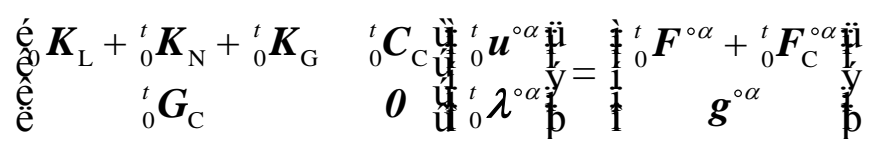

The sensitivity of cost function can be determined with the following expression

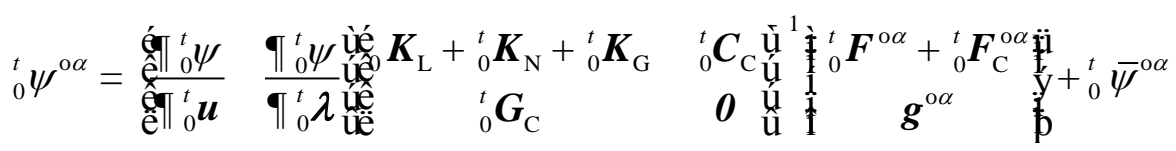

where $\frac{\mathbb{\coprod}_{0}^{t} \psi}{\prod_{0}^{t} \boldsymbol{\lambda}}=\boldsymbol{O}$ because the cost function does not directly depend on the traction at the contact surface.

Using the adjoint approach, let us put

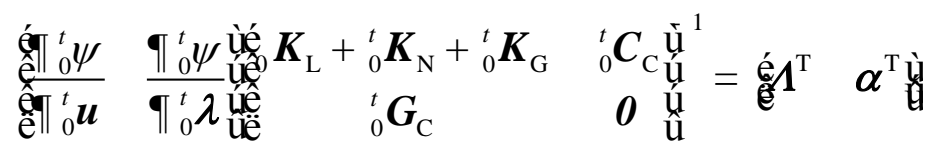

The adjoint equation system can hence be formulated as

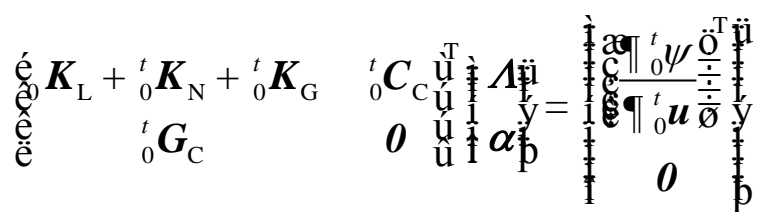

The matrix of coefficients of the adjoint equation system is the transpose of the original system of equations because the matrix of coefficients is not symmetric. Then, 


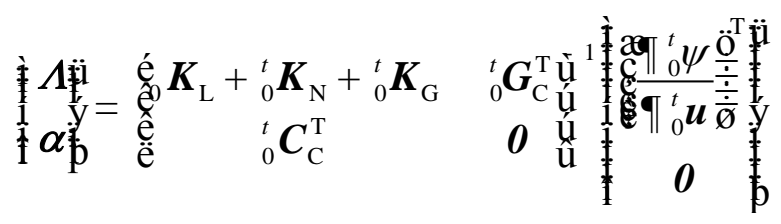

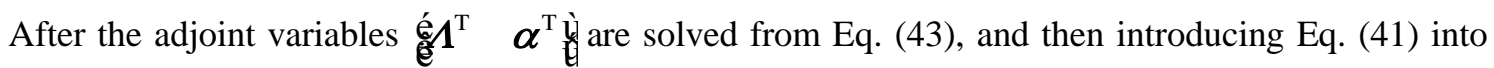
Eq. (40), the sensitivity of cost function is hence expressed as:

$$
{ }_{0}^{t} \psi^{\mathrm{o} \alpha}=\boldsymbol{\Lambda}^{\mathrm{T}}\left\{{ }_{0}^{t} \boldsymbol{F}^{\mathrm{o} \alpha}+{ }_{0}^{t} \boldsymbol{F}_{\mathrm{C}}^{\mathrm{o} \alpha}\right\}+\boldsymbol{\alpha}^{\mathrm{T}}\left\{\boldsymbol{g}^{\mathrm{o} \alpha}\right\}+{ }_{0}^{t} \bar{\psi}^{\mathrm{o} \alpha}
$$

For example, for the average strain to be determined in the belt end area of the tire - Eq. (1) - the cost function is

$$
\begin{aligned}
& { }_{\tau}^{t} \psi^{1}=\frac{1}{{ }_{\tau} V_{\mathrm{b}}} \int_{{ }_{\tau} V_{\mathrm{b}}}{ }_{\tau}^{t} \bar{\varepsilon}(\boldsymbol{x}) \mathrm{d}_{\tau} V \\
& { }_{\tau}^{t} \bar{\varepsilon}=\sqrt{\frac{2}{3}{ }_{\tau}^{t} e_{i j}{ }_{\tau}^{t} e_{i j}}=\sqrt{\frac{2}{3}\left({ }_{\tau}^{t} \varepsilon_{i j}-\frac{1}{3}{ }_{\tau}^{t} \varepsilon_{k k} \delta_{i j}\right)\left({ }_{\tau}^{t} \varepsilon_{i j}-\frac{1}{3}{ }_{\tau}^{t} \varepsilon_{l l} \delta_{i j}\right)}
\end{aligned}
$$

Sensitivities with respect to shape variables can be determined as follows

$$
\left({ }_{0}^{t} \Psi_{1}\right)^{\circ \alpha}=\Lambda^{\mathrm{T}}\left\{{ }_{0}^{t} \boldsymbol{F}^{\circ \alpha}+{ }_{0}^{t} \boldsymbol{F}_{\mathrm{C}}^{\circ \alpha}\right\}+\boldsymbol{\alpha}^{\mathrm{T}}\left\{\boldsymbol{g}^{\circ \alpha}\right\}+\left({ }_{0}^{t} \bar{\Psi}_{1}\right)^{\circ \alpha}
$$

If the design velocity is zero in a given region of design space, cost function will be insensitive to shape variations. In the adjoint system (43), the right side term is called adjoint load. In the present case, it can be computed as follows

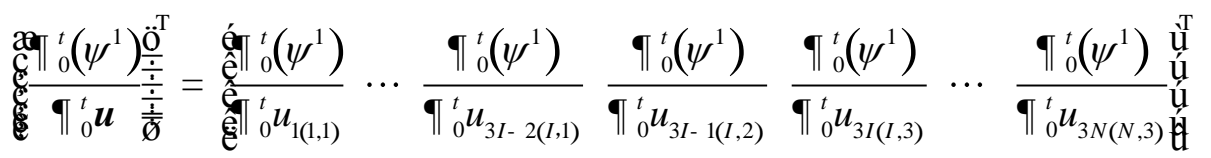

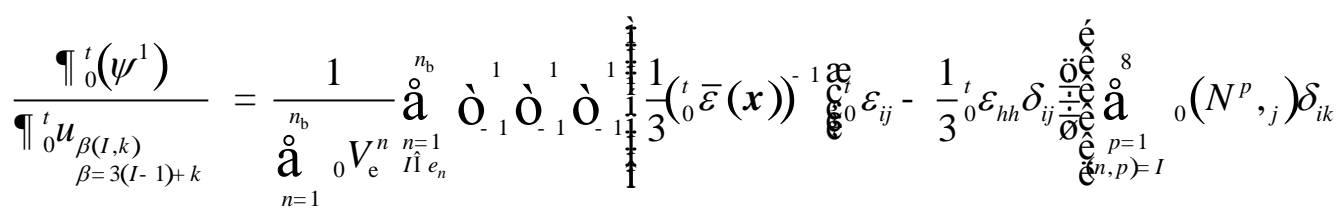

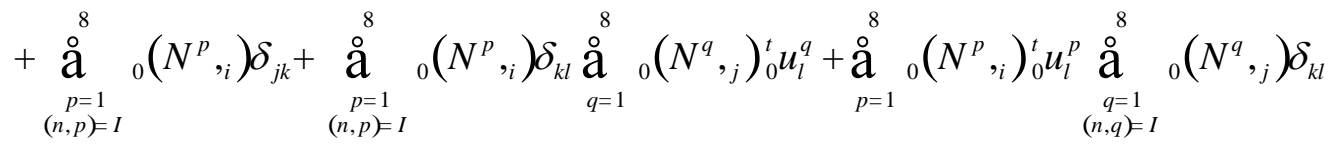

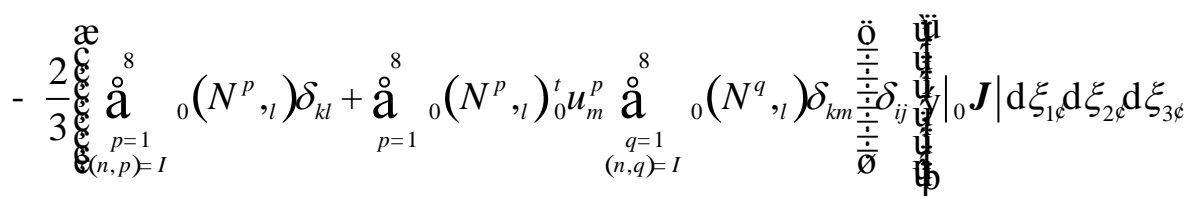




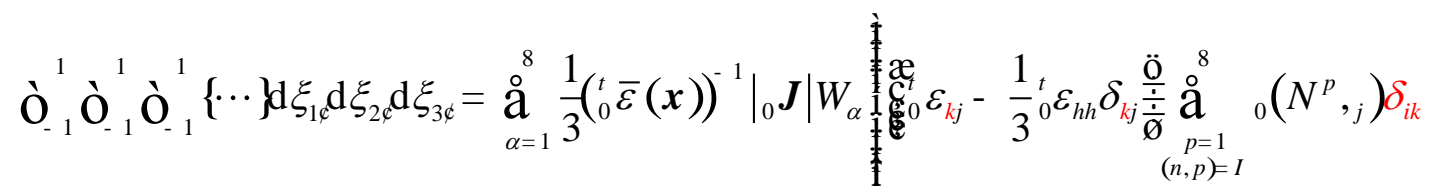

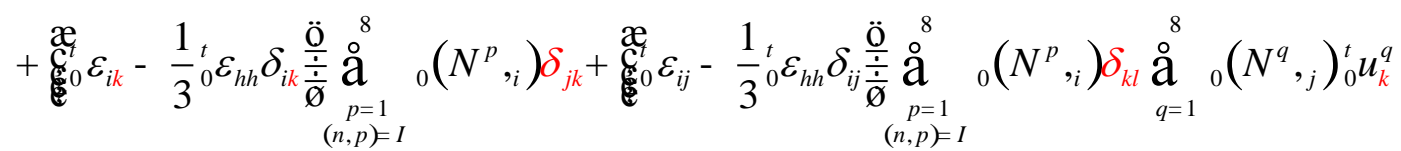

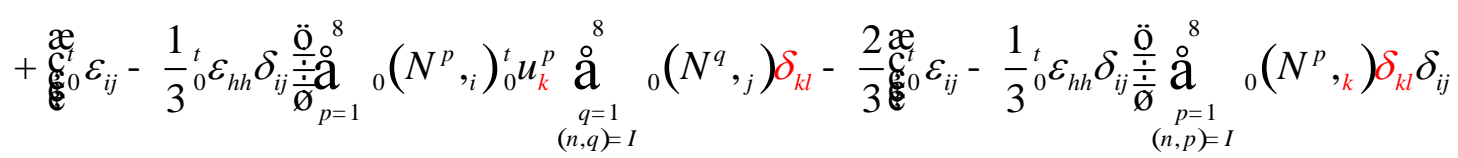

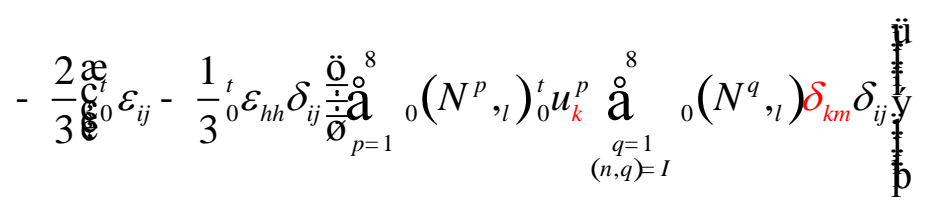

where the subscript $\beta$ of the displacement $u$ is the global number of the node displacement component for all nodes related to the cost function, which is denoted as $\beta(I, k)$ for the $I$-th node $k$-th degree of freedom, and takes the value $\beta=3(I-1)+k ; n$ is the number of element related to the cost function, the summation on all related boundary elements, number 1 to $n_{\mathrm{b}}$, the summation performed only on the elements connected to the $I$-th node; $q$ or $p$ is the local number of the element node, the condition $(n, p)=I$ under summation symbol means that, if the summation conducted only for the node in element $n$, coincides with the global $I$-th node; $N^{p}$ or $N^{q}$ denote the shape functions of the $p$-th or $q$-th element node.

In order to simplify Eq. (48), it can be written 


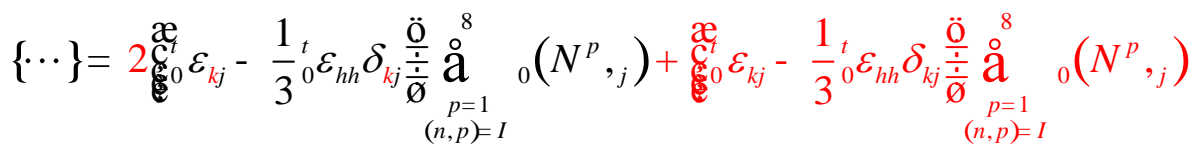

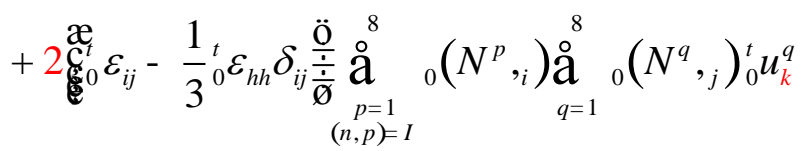

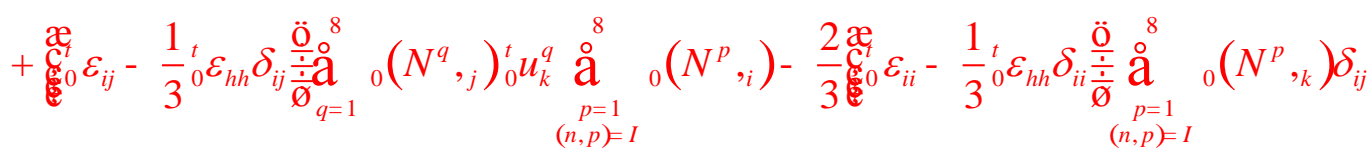

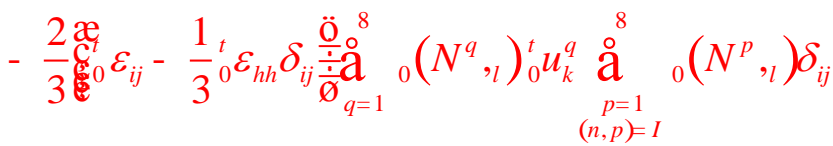

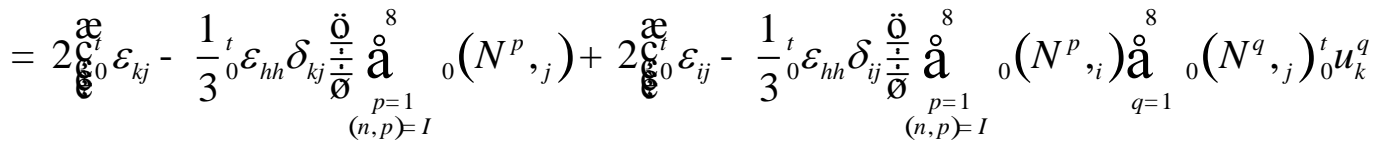

The terms indicated in red are eliminated.

In summary, the AVM requires to calculate the adjoint load and solve Eq. (43). Therefore, its computational cost is much lower than in the case of DDM if the number of optimization variables is larger than the number of objective functions.

\section{Determination of Sensitivities in the Shape Optimization of a Passenger Car Radial Tire}

The sensitivity analysis technique described in this study was integrated with a finite element program available to authors. Computational tasks are distributed as follows:

Stage 1: Compute boundary design velocities with a finite difference scheme

\section{Stage 2: Compute adjoint loads from Eq. (47)}

Stage 3: Determine adjoint variables from Eq. (43)

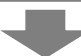

Stage 4: Compute fictitious loads and changing rate of real loads from Eq. (30)

\section{Stage 5: Compute sensitivities of cost function from} Eq. (44)
Subroutine: VSHAPE

Subroutines: CAL_ADJOINT_LOAD, STIFS_ADJ, STIFE8_ADJ, STIFE_ADJ

Subroutine: FRONT_IMP_ADJ

Subroutine: CAL_FICT_LOAD, STIFS_FICT, STIFE8_FICT, STIFE6_FICT, STIFE8R_FICT, STIFE_FICT

Subroutine: CAL_FICT_LOAD 
Sensitivity calculations were carried out for a 205/50R16 passenger radial car tire using both DDM and AVM. It was found that the two methods give identical results. However, the pure computational time of AVM (determined by subtracting the computation time required by the contact problem solver from the total CPU time required by the whole process) is only about $1 / 5$ of the CPU time required by DDM for evaluating sensitivities (see Table 3). DDM and AVM require almost the same time to solve the contact problem. However, because of the advantage of AVM mentioned in Section 7, the pure computational time of present approach can reduce to $1 / n$ of DDM, where $n$ is the number of design variables. Further discussion on this aspect is provided in Appendix 2. Sensitivity of cost function was evaluated with respect to the five shape variables included in the optimization problem. For that purpose, eight levels were evenly selected by Latin Hypercube Design method [3] in the range of $W_{1} \in[40.6,50.6], W_{2} \in[90.3,95.3], R_{1} \in[600,1200], R_{2} \in[248,600]$, and $R_{3} \in[40,53]$ covering the whole design range for all variables as shown in Table 1. According to orthogonal-maximin Latin Hypercube Design algorithm [4, 5], a number of samples greater than 1.5 times of the number of design variables is enough. Hence, the eight samples selected in the present analysis can efficiently cover the whole design range for five design variables.

The 2D and 3D models of the selected 205/55R16 passenger car radial tire are shown in Figure 2 and 4 , respectively. Table 2 presents the results of the sensitivity analysis carried out for eight design points generated with the design of experiments technique. $\psi_{A}^{\alpha}=\Lambda_{0}^{\mathrm{T} t} \boldsymbol{F}^{\mathrm{o} \alpha}, \psi_{B}^{\alpha}=\boldsymbol{\Lambda}_{0}^{\mathrm{T} t} \boldsymbol{F}_{\mathrm{C}}^{\mathrm{o} \alpha}$, $\psi_{C}^{\alpha}=\boldsymbol{\alpha}^{\mathrm{T}}\left\{\boldsymbol{g}^{\mathrm{o} \alpha}\right\}, \psi_{1}$ is the objective function, $\psi_{A V M}^{\alpha}=\psi_{A}^{\alpha}+\psi_{B}^{\alpha}-\psi_{C}^{\alpha}$ is the sensitivity value determined with the adjoint variable method, $\psi_{F D M}^{\alpha}$ is the sensitivity value determined with the finite differentiation method, and $\psi_{D D M}^{\alpha}$ is the sensitivity value determined with the direct differentiation method. It can be seen that AVM and DDM obtained identical values sensitivities while the FDM results show a slight error that may be caused by the fact that, unlike AVM and DDM, FDM computes sensitivity by directly changing design variables. In this study, the interval for design variable change was set as one thousand of the original variable value. 

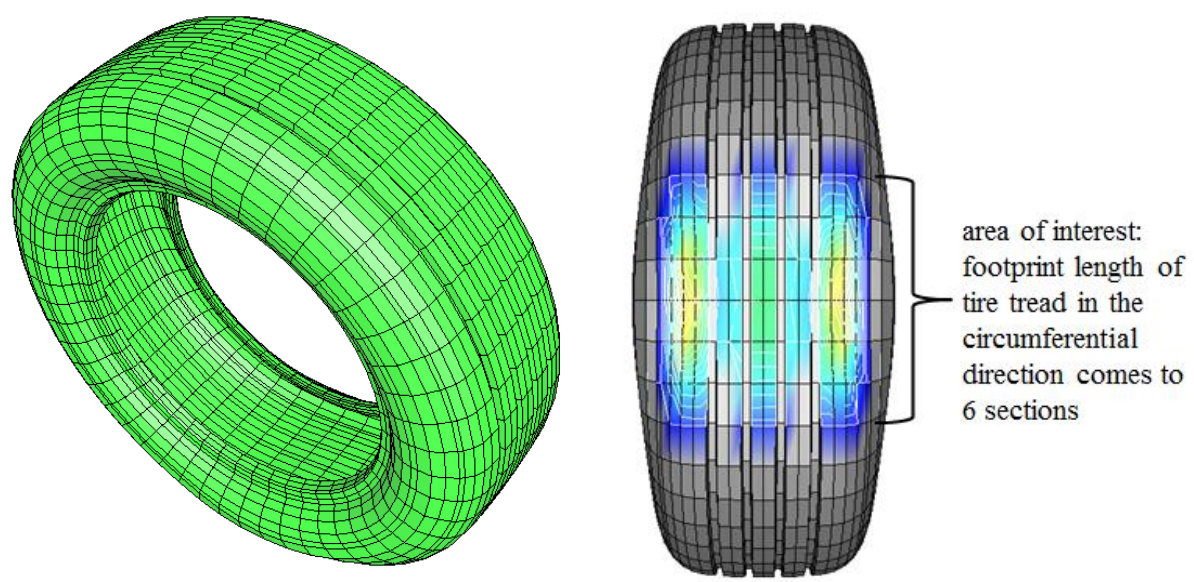

Figure 4: 3D finite element model of 205/65R16 tire

\section{Summary and Conclusions}

This paper described an efficient method for performing sensitivity analysis in shape optimization problems of complex nonlinear structures such as automotive tires. Since computations for each design sample are time consuming, reducing the number of design samples is very important. In shape optimization problems, two issues must be addressed: (i) sensitivity calculation and (ii) disposing of an efficient optimizer. A possible strategy may be to combine AVM and GBK. While the latter was already studied in the literature [4], this research developed a novel AVM including a new adjoint shape design sensitivity formulation for nonlinear structures subject to contact forces. Sensitivity equations of displacements with respect to shape variations were formulated using the virtual work principle. Then, sensitivity analysis of average shear strains in the tire belt area was carried out. It was found that the proposed approach is more accurate than traditional finite difference sensitivity computations and much faster than the direct differentiation method as shown in Table 3. In view of this, the present approach has full potentiality for becoming a useful framework for shape optimization and sensitivity analysis of highly nonlinear structures including contact forces. 
Table 1. Partitions of design space defined for the 205/55R16 tire shape optimization problem

$\begin{array}{cccccc} & W_{1} & W_{2} & R_{1} & R_{2} & R_{3} \\ \text { Level 1 } & 40.6 & 90.3 & 600 & 248 & 40 \\ \text { Level 2 } & 42.02857 & 91.01429 & 685.7143 & 298.2857 & 41.85714 \\ \text { Level 3 } & 43.45714 & 91.72857 & 771.4286 & 348.5714 & 43.71429 \\ \text { Level 4 } & 44.88571 & 92.44286 & 857.1429 & 398.8571 & 45.57143 \\ \text { Level 5 } & 46.31429 & 93.15714 & 942.8571 & 449.1429 & 47.42857 \\ \text { Level 6 } & 47.74286 & 93.87143 & 1028.571 & 499.4286 & 49.28571 \\ \text { Level 7 } & 49.17143 & 94.58571 & 1114.286 & 549.7143 & 51.14286 \\ \text { Level 8 } & 50.6 & 95.3 & 1200 & 600 & 53\end{array}$

Table 2. Results of sensitivity analysis carried out for the 205/55R 16 tire shape optimization problem

\begin{tabular}{|c|c|c|c|c|c|c|c|c|c|}
\hline $\begin{array}{c}\mathrm{DOE} \\
\text { sample }\end{array}$ & $\begin{array}{c}\text { Design } \\
\text { variables }\end{array}$ & $\begin{array}{c}\text { Value of } \\
\text { design } \\
\text { variables }\end{array}$ & $\psi_{A}^{\alpha}$ & $\psi_{B}^{\alpha}$ & $\psi_{C}^{\alpha}$ & $\psi_{1}$ & $\psi_{A V M}^{\alpha}$ & $\psi_{F D M}^{\alpha}$ & $\psi_{D D M}^{\alpha}$ \\
\hline \multirow{5}{*}{ \#1 } & 1 & $4.06 \mathrm{E}+01$ & $-1.46 \mathrm{E}-05$ & $-4.61 \mathrm{E}-07$ & $-9.23 \mathrm{E}-05$ & 4.99E-02 & 7.72E-05 & $7.44 \mathrm{E}-05$ & 7.72E-05 \\
\hline & 2 & $9.46 \mathrm{E}+01$ & $9.89 \mathrm{E}-07$ & $-2.63 \mathrm{E}-07$ & $7.55 \mathrm{E}-07$ & 4.99E-02 & $-2.77 \mathrm{E}-08$ & $-1.40 \mathrm{E}-06$ & $-2.77 \mathrm{E}-08$ \\
\hline & 3 & $1.03 \mathrm{E}+03$ & $-6.21 \mathrm{E}-07$ & $-1.30 \mathrm{E}-07$ & $-3.09 \mathrm{E}-06$ & 4.99E-02 & $2.34 \mathrm{E}-06$ & $2.33 \mathrm{E}-06$ & 2.34E-06 \\
\hline & 4 & $3.49 \mathrm{E}+02$ & $-6.98 \mathrm{E}-09$ & 7.80E-08 & $-3.92 \mathrm{E}-06$ & 4.99E-02 & $3.99 \mathrm{E}-06$ & $3.22 \mathrm{E}-06$ & $3.99 \mathrm{E}-06$ \\
\hline & 5 & $5.30 \mathrm{E}+01$ & $1.64 \mathrm{E}-04$ & $-1.21 \mathrm{E}-04$ & 4.54E-04 & 4.99E-02 & $-4.10 \mathrm{E}-04$ & $-1.18 \mathrm{E}-04$ & $-4.10 \mathrm{E}-04$ \\
\hline \multirow{5}{*}{$\# 2$} & 1 & $4.20 \mathrm{E}+01$ & $-3.10 \mathrm{E}-06$ & $-4.92 \mathrm{E}-07$ & $-5.82 \mathrm{E}-05$ & $4.84 \mathrm{E}-02$ & $5.46 \mathrm{E}-05$ & $5.89 \mathrm{E}-05$ & $5.46 \mathrm{E}-05$ \\
\hline & 2 & $9.32 \mathrm{E}+01$ & $-6.96 \mathrm{E}-05$ & 2.99E-05 & $1.01 \mathrm{E}-04$ & 4.84E-02 & $-1.41 \mathrm{E}-04$ & $-2.69 \mathrm{E}-04$ & $-1.40 \mathrm{E}-04$ \\
\hline & 3 & $6.00 \mathrm{E}+02$ & $-1.31 \mathrm{E}-06$ & $-6.50 \mathrm{E}-07$ & $-1.71 \mathrm{E}-05$ & 4.84E-02 & $1.52 \mathrm{E}-05$ & $1.72 \mathrm{E}-05$ & $1.52 \mathrm{E}-05$ \\
\hline & 4 & $4.49 \mathrm{E}+02$ & $1.37 \mathrm{E}-09$ & $1.88 \mathrm{E}-07$ & $-8.12 \mathrm{E}-06$ & 4.84E-02 & $8.31 \mathrm{E}-06$ & $8.33 \mathrm{E}-06$ & 8.31E-06 \\
\hline & 5 & $4.00 \mathrm{E}+01$ & $2.95 \mathrm{E}-04$ & $-7.20 \mathrm{E}-05$ & $-1.67 \mathrm{E}-04$ & 4.84E-02 & 3.89E-04 & $2.88 \mathrm{E}-04$ & 3.89E-04 \\
\hline \multirow{2}{*}{$\# 3$} & 1 & $4.35 \mathrm{E}+01$ & $-6.03 \mathrm{E}-06$ & $-1.02 \mathrm{E}-06$ & $-3.87 \mathrm{E}-05$ & 5.04E-02 & $3.17 \mathrm{E}-05$ & $3.27 \mathrm{E}-05$ & $3.17 \mathrm{E}-05$ \\
\hline & 2 & $9.03 \mathrm{E}+01$ & $-6.86 \mathrm{E}-07$ & $4.84 \mathrm{E}-06$ & $-1.11 \mathrm{E}-05$ & $5.04 \mathrm{E}-02$ & $1.53 \mathrm{E}-05$ & $-2.03 \mathrm{E}-05$ & $1.53 \mathrm{E}-05$ \\
\hline
\end{tabular}




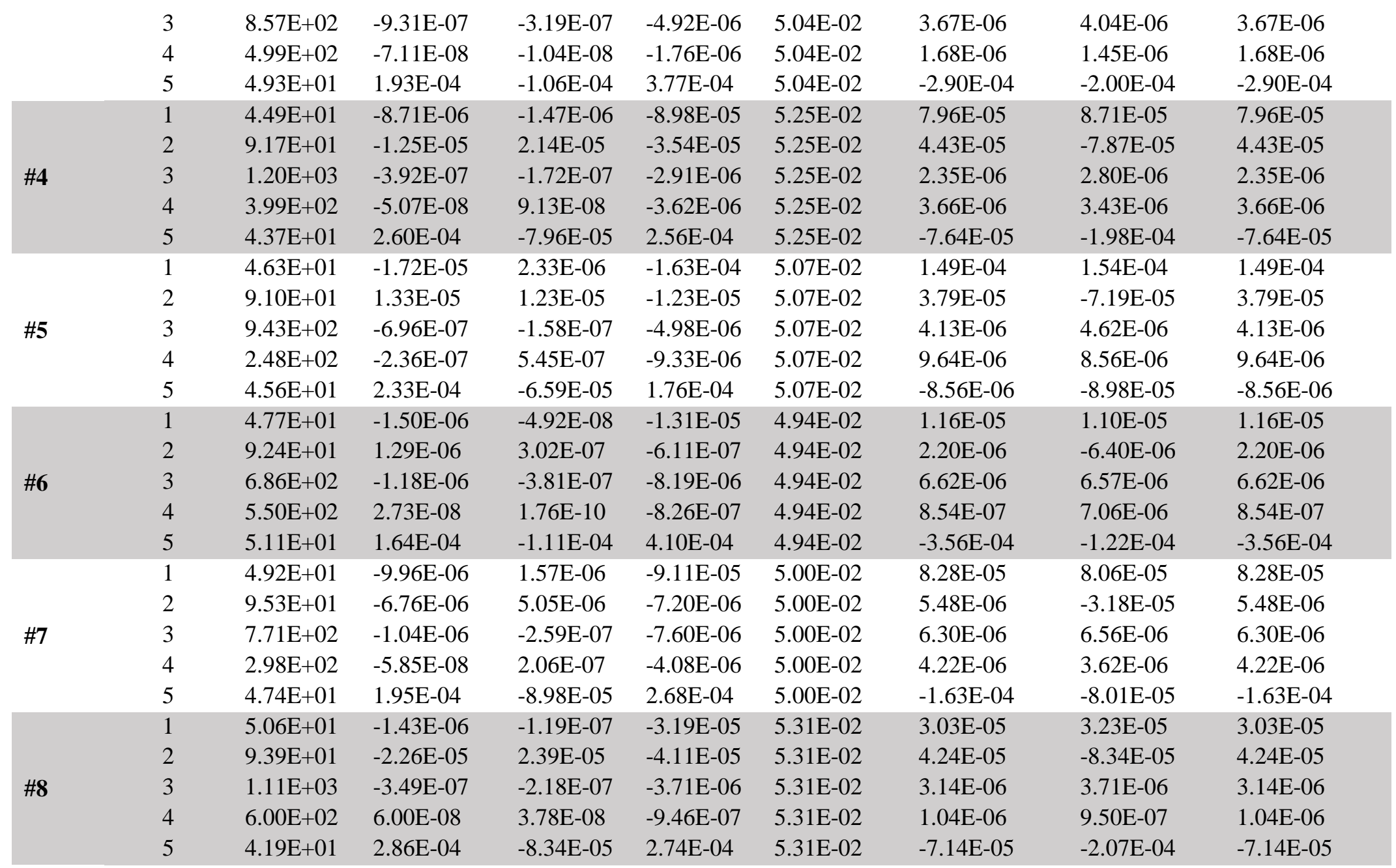


Table 3 Comparison of computing time of DDM and AVM (CPU time/seconds)

\begin{tabular}{|c|c|c|c|c|c|c|c|}
\hline \multirow{2}{*}{$\begin{array}{c}\text { DOE } \\
\text { sample }\end{array}$} & \multicolumn{3}{|c|}{ DDM } & \multicolumn{3}{|c|}{ AVM } & \multirow{2}{*}{$\begin{array}{l}\text { \% reduction of } \\
\text { CPU time } \\
\text { passing from } \\
\text { DDM to AVM }\end{array}$} \\
\hline & $\begin{array}{l}\text { Total } \\
\text { time }\end{array}$ & $\begin{array}{c}\text { Contact } \\
\text { solving } \\
\text { time }\end{array}$ & $\begin{array}{c}\text { DDM } \\
\text { algorithm } \\
\text { time }\end{array}$ & $\begin{array}{l}\text { Total } \\
\text { time }\end{array}$ & $\begin{array}{c}\text { Contact } \\
\text { solving } \\
\text { time }\end{array}$ & $\begin{array}{c}\text { AVM } \\
\text { algorithm } \\
\text { time }\end{array}$ & \\
\hline$\# 1$ & 430.50 & 341.05 & 89.45 & 359.22 & 339.64 & 19.58 & $78.11 \%$ \\
\hline$\# 2$ & 436.67 & 346.59 & 90.08 & 364.97 & 345.80 & 19.17 & $78.72 \%$ \\
\hline \#3 & 444.28 & 355.19 & 89.09 & 373.56 & 354.27 & 19.30 & $78.34 \%$ \\
\hline \#4 & 440.23 & 346.27 & 93.97 & 364.17 & 344.89 & 19.28 & $79.48 \%$ \\
\hline \#5 & 436.19 & 346.05 & 90.14 & 363.75 & 344.52 & 19.23 & $78.66 \%$ \\
\hline \#6 & 440.42 & 348.83 & 91.59 & 366.13 & 347.11 & 19.02 & $79.24 \%$ \\
\hline \#7 & 440.59 & 351.38 & 89.22 & 369.03 & 349.72 & 19.31 & $78.35 \%$ \\
\hline$\# 8$ & 437.98 & 348.38 & 89.61 & 366.42 & 346.70 & 19.72 & $78.00 \%$ \\
\hline
\end{tabular}




\section{Acknowledgements}

Projects 11672148,51275265 and 5117528 were supported by the National Science Foundation of China. This study was partially supported by the Manufacture Française des Pneumatiques Michelin and AvH Foundation. Valuable comments from three reviewers are greatly appreciated.

\section{References}

1. van Keulen F, Haftka RT, Kim NH. Review of options for structural design sensitivity analysis, 1: linear systems. Computer Methods in Applied Mechanics and Engineering, 2005; 194(30-33): 32133243.

2. Wei Y, Rezgui A, Yao Z, Wang P. A comparative analysis of contact algorithms in contact shape optimization problems. Optimization and Engineering, 2012; 13(4): 595-623.

3. Xuan Y, Xiang J, Zhang W, Zhang Y. Gradient-based Kriging approximate model and its application research to optimization design. Science in China E, 2009; 52: 1117-1124.

4. Yao Z, Wei Y. Some ideas and progress on the shape optimization of nonlinear structures. Procedia Engineering, 2012; 31: 600-607.

5. Joesph VR, Hung Y. Orthogonal-maximin Latin hypercube designs. Statistica Sinica, 2008; 18: 171186.

6. Choi KK, Kim NH. Structural Sensitivity Analysis and Optimization, 1: Linear Systems. 2005; Berlin: Springer.

7. Choi KK, Kim NH. Structural Sensitivity Analysis and Optimization, 2: Nonlinear Systems and Applications. 2005, Berlin: Springer.

8. Burczynski T, Kane JH, Balakrishna C. Shape design sensitivity analysis via material derivativeadjoint variable technique for 3-D and 2-D curved boundary elements. International Journal for Numerical Methods in Engineering, 1995; 38(17): 2839-2866.

9. Burczynski T, Kane JH, Balakrishna C. Comparison of shape design sensitivity analysis formulations via material derivative-adjoint variable and implicit differentiation techniques for 3-D and 2-D curved boundary element. Computer Methods in Applied Mechanics and Engineering, 1997; 142(1-2): 89-109.

10. Cho S, Choi KK. Design sensitivity analysis and optimization of non-linear transient dynamics, I: sizing design. International Journal for Numerical Methods in Engineering, 2000; 48(3): 351-373.

11. Choi KK, Duan W. Design sensitivity analysis and shape optimization of structural components with hyperelastic material. Computer Methods in Applied Mechanics and Engineering, 2000; 187(1-2): 219-243.

12. Grindeanu I, Chang KH, Choi KK et al. Design sensitivity analysis of hyperelastic structures using a meshless method. AIAA Journal, 1998; 36(4): 618-627.

13. Hou GJW, Sheen JS, Chuang CH. Shape-sensitivity analysis and design optimization of linear, thermoelastic solids. AIAA Journal, 1992; 30(2): 528-537.

14. Meric RA. Shape design sensitivity analysis and optimization for nonlinear heat and electric conduction problems. Numerical Heat Transfer Part a-Applications, 1998; 34(2): 185-203.

15. Park IH, Coulomb JL, Hahn SY. Design sensitivity analysis for nonliner magnetostatic problems by continuum approach. Journal de Physique III, 1992; 2(11): 2045-2053.

16. Park IH, Lee HB, Kwak IG et al. Design sensitivity analysis for steady-state eddy-current problems by continuum approach. IEEE Transactions on Magnetics, 1994; 30(5): 3411-3414.

17. Park JS, Choi KK. Design sensitivity analysis and optimization of nonlinear structural systems with critical loads. Journal of Mechanical Design, 1992; 114(2): 305-312. 
18. Ryu JS, Yao YY, Koh CS et al. Optimal shape design of 3-D nonlinear electromagnetic devices using parameterized design sensitivity analysis. IEEE Transactions on Magnetics, 2005; 41(5): 1792-1795.

19. Santos JLT, Choi KK. Shape design sensitivity analysis of nonlinear structural systems. Structural Optimization, 1992; 4(1): 23-35.

20. Smith DE, Tortorelli DA, Tucker CL. Optimal design for polymer extrusion, II: sensitivity analysis for weakly-coupled nonlinear steady-state systems. Computer Methods in Applied Mechanics and Engineering, 1998; 167(3-4): 303-323.

21. Moon MY, Kim JH, Ha YD et al. Adjoint design sensitivity analysis of dynamic crack propagation using peridynamic theory. Structural and Multidisciplinary Optimization, 2015; 51(3): 585-598.

22. Jang HL, Kim JH, Park Y et al. Adjoint design sensitivity analysis of molecular dynamics in parallel computing environment. International Journal of Mechanics and Materials in Design, 2014; 10(4): 379394.

23. Jang HL, Cho S. Adjoint shape design sensitivity analysis of fluid-solid interactions using concurrent mesh velocity in ALE formulation. Finite Elements in Analysis and Design, 2014; 85: 20-32.

24. Kim MG, Jang H, Kim $\mathrm{H}$ et al. Multiscale adjoint design sensitivity analysis of atomistic-continuum dynamic systems using bridging scale decomposition. Modelling and Simulation in Materials Science and Engineering, 2013; 21(3): 035005(25pp).

25. Matsumoto $\mathrm{T}$, Yamada $\mathrm{T}$, Takahashi $\mathrm{T}$ et al. Acoustic design shape and topology sensitivity formulations based on adjoint method and BEM. CMES-Computer Modeling in Engineering \& Sciences, $2011 ; \mathbf{7 8}(2)$ : 77-94.

26. Igarashi $\mathrm{H}$, Watanabe K. Complex adjoint variable method for finite-element analysis of eddy current problems. IEEE Transactions on Magnetics, 2010; 46(8): 2739-2742.

27. Lee TH. Adjoint method for design sensitivity analysis of multiple eigenvalues and associated eigenvectors. AIAA Journal, 2007; 45(8): 1998-2004.

28. Thomas JP, Hall KC, Dowell EH. Discrete adjoint approach for modeling unsteady aerodynamic design sensitivities. AIAA Journal, 2005; 43(9): 1931-1936.

29. Martins J, Alonso JJ, Reuther JJ. A coupled-adjoint sensitivity analysis method for high-fidelity aerostructural design. Optimization and Engineering, 2005; 6(1): 33-62.

30. Maute K, Nikbay M, Farhat C. Sensitivity analysis and design optimization of three-dimensional nonlinear aeroelastic systems by the adjoint method. International Journal for Numerical Methods in Engineering, 2003; 56(6): 911-933.

31. Georgieva NK, Glavic S, Bakr MH et al. Feasible adjoint sensitivity technique for EM design optimization. IEEE Transactions on Microwave Theory and Techniques, 2002; 50(12): 2751-2758.

32. Lee TH. An adjoint variable method for design sensitivity analysis of elastoplastic structures. KSME International Journal, 1999; 13(3): 246-252.

33. Park IH. Design sensitivity analysis for transient eddy current problems using finite element discretization and adjoint variable. IEEE Transactions on Magnetics, 1996; 32(3): 1242-1245.

34. Belegundu AD, Arora JS. A sensitivity interpretation of adjoint variables in optimal-design. Computer Methods in Applied Mechanics and Engineering, 1985; 48(1): 81-89.

35. Guz AN, Fundamentals of the Three-Dimensional Theory of Stability of Deformable Bodies, SpringerVerlag Berlin Heidelberg 1999. 


\section{Appendix 1}

\begin{tabular}{|c|c|}
\hline Notation & \\
\hline${ }_{\tau}^{t} \psi^{1}$ & The objective function \\
\hline$\tau$ & The initial configuration corresponding to the shape parameter \\
\hline$t$ & The load parameter \\
\hline$e_{i j}$ & The principle strains \\
\hline$\delta_{i j}$ & The Kronecker delta \\
\hline${ }_{\tau} V_{b}$ & The total volume of the design area \\
\hline${ }_{\tau}^{t} \bar{\varepsilon}(\boldsymbol{x})$ & The effective shear strain \\
\hline${ }_{0} x_{i},{ }_{\tau}^{0} x_{i}$ & $\begin{array}{l}\text { The Lagrange coordinates for the original shape and the shape design corresponding to } \\
\text { parameters } \tau_{\alpha}\end{array}$ \\
\hline$m$ & The number of independent degrees of freedom (DOFs) \\
\hline$k$ & The number of design parameters \\
\hline${ }_{\tau}^{0} u_{i}$ & Virtual displacement \\
\hline${ }_{0}^{t} u_{i}^{\circ \alpha}$ & The sensitivity of the displacements when $\tau_{\alpha}=0$ \\
\hline${ }_{0}^{t}\left(u_{i},{ }_{j}\right)^{\circ \alpha}$ & The sensitivity of displacement gradients \\
\hline${ }_{0} V_{i \alpha}$ & Shape design velocity field \\
\hline$\tau_{\alpha}$ & The design variables \\
\hline$\Delta \tau_{\alpha}$ & A small increment of a design variable \\
\hline$B, D$ & The tire width and diameter \\
\hline$\Omega$ & The whole shape domain \\
\hline$x_{o i}, y_{o i}$ & The coordinates of the $i^{t h}$ arc center \\
\hline$x_{i}, y_{i}$ & The outer surface point coordinates belonging to the $i^{t h}$ arc \\
\hline$T$ & Mapping function \\
\hline$u$ & The parametric coordinates \\
\hline $\mathbf{F}, \mathbf{B}$ & The coefficient matrices \\
\hline $\mathbf{R}$ & The vector of design variables \\
\hline${ }_{\tau}^{t} C_{c}^{\mathrm{LM}}$ & The contact boundary term in Lagrange multiplier approach \\
\hline${ }_{0} \lambda_{n},{ }_{\tau}^{t} \lambda_{n}$ & The Lagrange multipliers \\
\hline${ }_{\tau}^{t} S^{\mathrm{f}},{ }_{\tau} S^{\mathrm{t}},{ }_{\tau}^{t} S^{\mathrm{st}}$ & The traction-free boundary, the traction-given boundary, and the contacted boundary \\
\hline${ }_{0}^{t} S_{i j}$ & The stress field \\
\hline${ }_{0}^{t} t_{k},{ }_{0} \rho_{0}^{t} f_{k}$ & The loads which are in equilibrium in the original shape \\
\hline${ }_{0} \boldsymbol{K}_{\mathrm{L}},{ }_{0}^{t} \boldsymbol{K}_{\mathrm{N}},{ }_{0}^{t} \boldsymbol{K}_{\mathrm{G}}$ & $\begin{array}{l}\text { Identical to those in the conventional nonlinear finite element analysis for the original } \\
\text { design shape }\end{array}$ \\
\hline${ }_{0}^{t} \boldsymbol{Q}^{\circ \alpha},{ }_{0}^{t} \boldsymbol{F}_{\mathrm{F}}^{\circ \alpha}$ & The change rates of the real loads and the fictitious loads \\
\hline
\end{tabular}




\begin{tabular}{ll}
\hline${ }_{0}^{t} \boldsymbol{F}_{\mathrm{F}}^{\circ \alpha}$ & The change rate of the fictitious load \\
${ }_{0}^{t} \boldsymbol{F}_{\mathrm{R}}^{\circ \alpha}$ & The change rate of the real loads \\
${ }_{0}^{t} \boldsymbol{u}^{\mathrm{o} \alpha}$ & Displacement sensitivities \\
$\psi_{A V M}^{\alpha}$ & The sensitivity value determined with the adjoint variable method \\
$\psi_{F D M}^{\alpha}$ & The sensitivity value determined with the finite differentiation method \\
$\psi_{D D M}^{\alpha}$ & The sensitivity value determined with the direct differentiation method \\
\hline
\end{tabular}

\section{Appendix 2: Calibration case}

In order to generalize the findings presented in this study, FDM, DDM, and AVM are applied to the square rod problem schematized in Figure A2.1. The top of rod is subject to uniform pressure while the bottom is put in frictionless contact with a stationary rigid surface. The rod is divided into 10 cubic HEX8 elements with length of $10 \mathrm{~mm}$ (see Figure A2.1). For the sake of simplicity, the side length $l_{c}$ of the first element is defined as design variable, closely related to nodal forces of top element. We assume that all four sides displace inwards or outwards together in a length $d$ (see Figure A2.1). The objective function is the effective shear strain -Eq. (2)- developed in the ninth element. The shape design velocity is 0.5 , i.e. the coordinate $x_{c}$ change rate with respect to the $l_{c}$ design variable. One can calculate the design sensitivities $\psi_{F D M}^{\alpha}, \psi_{D D M}^{\alpha}, \psi_{A V M}^{\alpha}$ and the corresponding CPU times listed in Table A2.1.

Similar to Table 2, AVM and DDM get identical sensitivity values while FDM shows a small error. For this simple case, AVM is only $22 \%$ faster than DDM. Hence, the advantage of using AVM becomes much more significant for large scale problems.

Table A2.1 Comparison of FDM, DDM, and AVM results for the square rod problem

\begin{tabular}{cccc}
\hline & FDM & DDM & AVM \\
\hline CPU time (sec) & 2.80 & 3.25 & 2.55 \\
Sensitivity of objective function & $2.66 \mathrm{E}-02$ & $2.61 \mathrm{E}-02$ & $2.61 \mathrm{E}-02$ \\
\hline
\end{tabular}




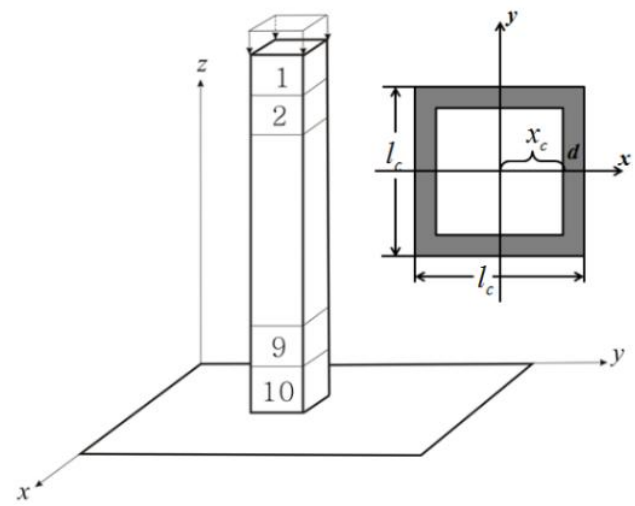

Figure A2.13D finite element model and boundary conditions of the square rod.

\section{Appendix 3: Derivation of strain field sensitivity equation Eq. (18)}

According to the nonlinear theory, the strain field can be expressed as follows:

$$
\begin{aligned}
& { }_{0}^{t} \varepsilon_{i j}=\left({ }_{0}^{t} u_{i},{ }_{j}+{ }_{0}^{t} u_{j},{ }_{i}+{ }_{0}^{t} u_{k},{ }_{i}{ }_{0} u_{k},{ }_{j}\right) / 2 \\
& { }_{\tau}^{t} \varepsilon_{i j}=\left({ }_{\tau}^{t} u_{i},{ }_{j}+{ }_{\tau}^{t} u_{j},{ }_{i}+{ }_{\tau}^{t} u_{k},{ }_{i}^{t} u_{k},{ }_{j}\right) / 2=\left({ }_{0}^{t} u_{i},{ }_{j}+{ }_{0}^{t} u_{j},{ }_{i}+{ }_{0}^{t} u_{k},{ }_{i}{ }_{i} u_{k},{ }_{j}\right) / 2 \\
& +\left({ }_{0}^{t}\left(u_{i},{ }_{j}\right)^{\circ \alpha}+{ }_{0}^{t}\left(u_{j},{ }_{i}\right)^{\circ \alpha}+{ }_{0}^{t}\left(u_{k},{ }_{i}\right)^{\circ \alpha}{ }_{0}^{t} u_{k},{ }_{j}+{ }_{0}^{t} u_{k},{ }_{i}^{t}{ }_{0}\left(u_{k},{ }_{j}\right)^{\circ \alpha}\right) \tau_{\alpha} / 2 \\
& +\left({ }_{0}^{t}\left(u_{k},{ }_{i}\right)^{\circ \alpha}{ }_{0}\left(u_{k},{ }_{j}\right)^{\circ \beta}\right) \tau_{\alpha} \tau_{\beta} / 2
\end{aligned}
$$

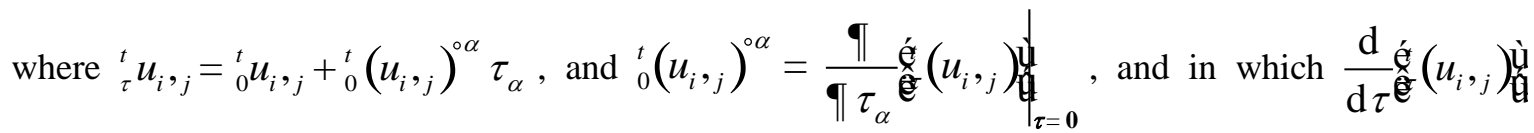
consists of two parts: one is due to the difference of ${ }_{\tau}^{t} u_{i}$ and ${ }_{0}^{t} u_{i}$, namely ${ }_{0}^{t} u_{i}^{\circ \alpha}$, another one comes from the difference of ${ }_{\tau}^{t}\left(u_{i},{ }_{j}\right)$ and ${ }_{0}^{t}\left(u_{i},{ }_{j}\right)$ in the case of identical ${ }_{\tau}^{t} u_{i}$ and ${ }_{0}^{t} u_{i}$.

For the virtual displacement $\delta_{\tau} u_{i}$, which is arbitrary and geometrically admissible for the design shape vector $\bar{\tau}$ including all shape variables, the corresponding virtual strain can be expressed as follows

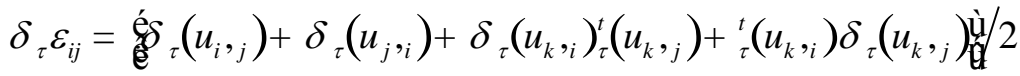

$$
\begin{aligned}
& =\left(\delta_{0} u_{i},{ }_{j}+\delta_{0} u_{j},_{i}+\delta_{0} u_{k},{ }_{i}{ }^{t} u_{k},{ }_{j}+{ }_{0}^{t} u_{k},{ }_{i} \delta_{0} u_{k},{ }_{j}\right) / 2 \\
& +\left(\delta_{0}\left(u_{i},{ }_{j}\right)^{\circ \alpha}+\delta_{0}\left(u_{j},{ }_{i}\right)^{\circ \alpha}+\delta_{0}\left(u_{k},{ }_{i}\right)^{\circ \alpha}{ }_{0} u_{k},{ }_{j}+{ }_{0}^{t}\left(u_{k},{ }_{i}\right)^{\circ \alpha} \delta_{0} u_{k},{ }_{j}+\delta_{0} u_{k},{ }_{i}{ }_{0}^{t}\left(u_{k},{ }_{j}\right)^{\circ \alpha}\right. \\
& \left.+{ }_{0}^{t} u_{k},{ }_{i} \delta_{0}\left(u_{k},{ }_{j}\right)^{\circ \alpha}\right) \tau_{\alpha} / 2+\left(\delta_{0}\left(u_{k},{ }_{i}\right)^{\circ \alpha}{ }_{0}^{t}\left(u_{k},{ }_{j}\right)^{\circ \beta}+{ }_{0}^{t}\left(u_{k},{ }_{i}\right)^{\circ \alpha} \delta_{0}\left(u_{k},{ }_{j}\right)^{\circ \beta}\right) \tau_{\alpha} \tau_{\beta} / 2
\end{aligned}
$$


By omitting the small higher order term $\left(\delta_{0}\left(u_{k},{ }_{i}\right)^{\circ \alpha}{ }_{0}\left(u_{k},{ }_{j}\right)^{\circ \beta}+{ }_{0}^{t}\left(u_{k},{ }_{i}\right)^{\circ \alpha} \delta_{0}\left(u_{k},{ }_{j}\right)^{\circ \beta}\right) \tau_{\alpha} \tau_{\beta} / 2$ which implies that the shape variation is small terms, the strain sensitivities can be rewritten as

$$
\begin{aligned}
\delta_{\tau} \varepsilon_{i j}= & \left(\delta_{0} u_{i},{ }_{j}+\delta_{0} u_{j},{ }_{i}+\delta_{0} u_{k},{ }_{i}{ }_{0} u_{k},{ }_{j}+{ }_{0}^{t} u_{k},{ }_{i} \delta_{0} u_{k},{ }_{j}\right) / 2 \\
+ & \left(\delta_{0}\left(u_{i},{ }_{j}\right)^{\circ \alpha}+\delta_{0}\left(u_{j},{ }_{i}\right)^{\circ \alpha}+\delta_{0}\left(u_{k},{ }_{i}\right)^{\circ \alpha}{ }_{0} u_{k},{ }_{j}+{ }_{0}^{t}\left(u_{k},{ }_{i}\right)^{\circ \alpha} \delta_{0} u_{k},{ }_{j}\right. \\
& \left.+\delta_{0} u_{k},{ }_{i}{ }_{0}\left(u_{k},{ }_{j}\right)^{\circ \alpha}+{ }_{0}^{t} u_{k},{ }_{i} \delta_{0}\left(u_{k},{ }_{j}\right)^{\circ \alpha}\right) \tau_{\alpha} / 2
\end{aligned}
$$

\section{Appendix 4: Derivation of displacement gradient sensitivity Eq. (23)}

Introducing Eqs (18)-(20) into the equation of virtual work corresponding to the shape variation, it follows

$$
\begin{aligned}
& \grave{\mathrm{O}}_{{ }_{0} V}{ }_{\tau}^{t} S_{i j} \delta_{\tau}^{t} \varepsilon_{i j}\left|{ }_{0}^{\tau} \boldsymbol{J}\right| \mathrm{d}_{0} V=\grave{\mathrm{O}}_{0 S^{t}}{ }_{\tau}^{t} t_{k} \delta_{\tau}^{t} u_{k}\left(\mathrm{~d}_{\tau} S / \mathrm{d}_{0} S\right) \mathrm{d}_{0} S+\grave{\mathrm{O}}_{{ }_{0} V}{ }_{\tau} \rho_{\tau}^{t} f_{k} \delta_{\tau}^{t} u_{k}\left|{ }_{0}^{\tau} J\right| \mathrm{d}_{0} V \\
& +\grave{\mathbf{O}}_{0} S_{c}^{\mathrm{st}}\left({ }_{\tau}^{t} \lambda_{n} \delta_{0} u_{n}+{ }_{\tau}^{t} \lambda_{s} \delta_{0} u_{s}+{ }_{\tau}^{t} \lambda_{t} \delta_{0} u_{t}\right)\left(\mathrm{d}_{\tau} S / \mathrm{d}_{0} S\right) \mathrm{d}_{0} S
\end{aligned}
$$

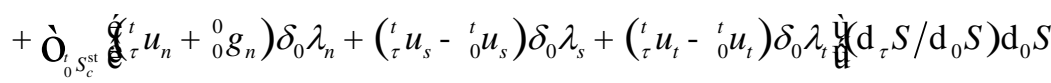

$$
\begin{aligned}
& +\grave{\mathrm{O}}_{0} S_{c}^{\mathrm{sl}}\left({ }_{\tau}^{t} \lambda_{n} \delta_{0} u_{n}+{ }_{\tau}^{t} t_{s} \delta_{0} u_{s}+{ }_{\tau}^{t} t_{t} \delta_{0} u_{t}\right)\left(\mathrm{d}_{\tau} S / \mathrm{d}_{0} S\right) \mathrm{d}_{0} S \\
& \left.+\grave{\mathrm{O}}_{0} S_{c}^{\mathrm{s}} \dot{q}_{\tau}^{t} u_{n}+{ }_{0}^{0} g_{n}\right) \delta_{0} \lambda_{n} \grave{̀}_{\mathrm{e}}\left(\mathrm{d}_{\tau} S / \mathrm{d}_{0} S\right) \mathrm{d}_{0} S
\end{aligned}
$$

The above equation can be rewritten as

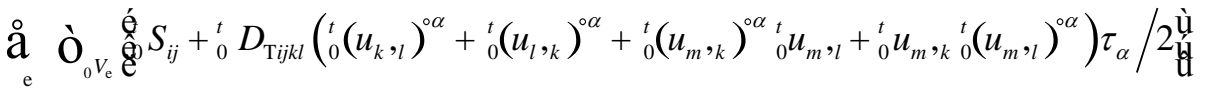

$$
\begin{aligned}
& \left.\times \delta_{0}^{\prime} \delta_{i} u_{j}+\delta_{0} u_{j},{ }_{i}+\delta_{0} u_{k},{ }_{i}{ }_{i} u_{k},{ }_{j}+{ }_{0}^{t} u_{k},{ }_{i} \delta_{0} u_{k},{ }_{j}\right) / 2+\left(\delta_{0}\left(u_{i},{ }_{j}\right)^{\circ \alpha}+\delta_{0}\left(u_{j},{ }_{i}\right)^{\circ \alpha}\right. \\
& \left.+\delta_{0}\left(u_{k},{ }_{i}\right)^{\circ \alpha}{ }_{0} u_{k},{ }_{j}+{ }_{0}^{t}\left(u_{k},{ }_{i}\right)^{\circ \alpha} \delta_{0} u_{k},{ }_{j}+\delta_{0} u_{k},{ }_{i}^{t}{ }_{0}\left(u_{k},{ }_{j}\right)^{\circ \alpha}+{ }_{0}^{t} u_{k},{ }_{i} \delta_{0}\left(u_{k},{ }_{j}\right)^{\circ \alpha}\right) \tau_{\alpha} / 2 \mathrm{ŭu}_{\mathrm{u}}^{\grave{u}}\left(1+{ }_{0} V_{i \alpha},{ }_{i} \tau_{\alpha}\right) \mathrm{d}_{0} V_{\mathrm{e}}
\end{aligned}
$$

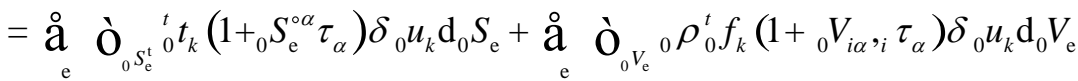

$$
\begin{aligned}
& +\underset{\mathrm{e}}{\stackrel{\circ}{\mathrm{O}_{t} S_{c}^{\mathrm{st}}}}\left({ }_{0}^{t} \lambda_{n}+{ }_{0}^{t} \lambda_{n}^{\circ \alpha} \tau_{\alpha}\right) \delta_{0} u_{n}\left(1+{ }_{0} S_{\mathrm{e}}^{\circ \beta} \tau_{\beta}\right) \mathrm{d}_{0} S_{\mathrm{e}} \\
& +\underset{\mathrm{e}}{\stackrel{\circ}{\mathrm{O}_{0}}} S_{c}^{\mathrm{st}}\left({ }_{0}^{t} u_{n}+{ }_{0}^{t} u_{n}^{\circ \alpha} \tau_{\alpha}+{ }_{0}^{0} g_{n}\right) \delta_{0} \lambda_{n}\left(1+{ }_{0} S_{\mathrm{e}}^{\circ \beta} \tau_{\beta}\right) \mathrm{d}_{0} S_{\mathrm{e}}
\end{aligned}
$$


In order to simplify the present derivation, only frictionless contact is considered here. In Eq. (A4.2), $S_{c}^{s t}$ denotes the contact element. Developing equation (A4.2) with respect to the shape variation factor $\tau_{\alpha}$, we have

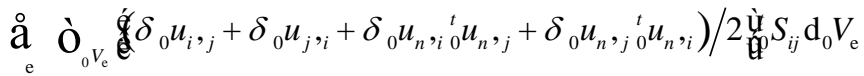

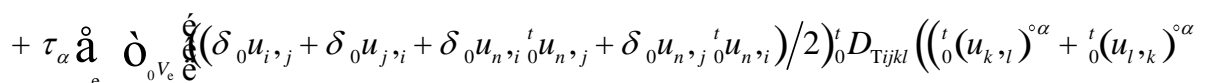

$$
\begin{aligned}
& \left.\left.+{ }_{0}^{t} u_{m},{ }_{0}^{t}\left(u_{m}, l\right)^{\circ \alpha}+{ }_{0}^{t} u_{m},{ }_{0}^{t}\left(u_{m},{ }_{k}\right)^{\circ \alpha}\right) / 2\right)_{\mathrm{u}}^{\grave{\mathrm{u}}_{\mathrm{d}}} \mathrm{d}_{0} V_{\mathrm{e}} \\
& +\tau_{\alpha} \stackrel{\circ}{\mathrm{a}} \grave{\mathrm{O}}_{0} V \dot{S}_{\mathrm{e}} \delta_{0}\left(u_{i},{ }_{j}\right)^{\circ \alpha}+\delta_{0}\left(u_{j},_{i}\right)^{\circ \alpha}+\delta_{0}\left(u_{n},{ }_{i}\right)_{0}^{t}\left(u_{n},{ }_{j}\right)^{\circ \alpha}+\delta_{0}\left(u_{n},{ }_{j}\right)_{0}^{t}\left(u_{n},_{i}\right)^{\circ \alpha}
\end{aligned}
$$

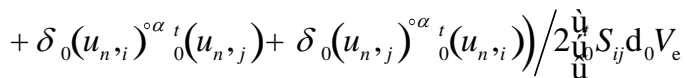

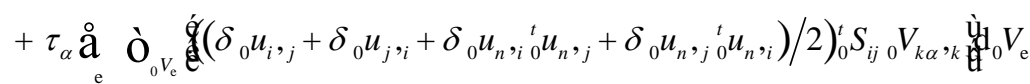

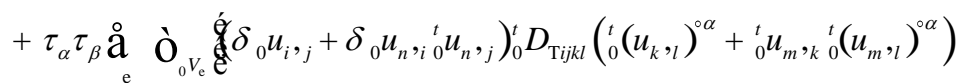

$$
\begin{aligned}
& +\left(\delta_{0}\left(u_{i},{ }_{j}\right)^{\circ \alpha} \alpha+\delta_{0}\left(u_{n},{ }_{i}\right)_{0}^{t}\left(u_{n},{ }_{j}\right)^{\circ \alpha} \alpha+\delta_{0}\left(u_{n},,_{i}\right)^{\circ \alpha} \alpha_{0}^{t}\left(u_{n},{ }_{j}\right)\right)_{0}^{t} S_{i j} \underset{\mathrm{u}}{\grave{\mathrm{u}}} V_{s \beta}, \mathrm{d}_{0} V_{\mathrm{e}}
\end{aligned}
$$

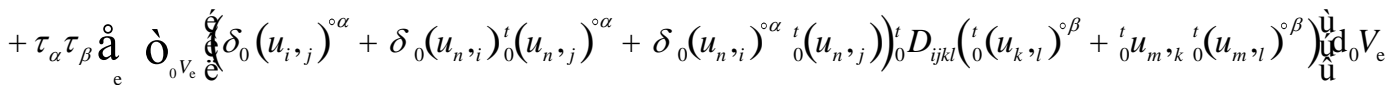

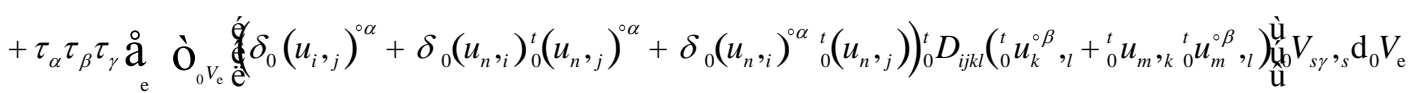

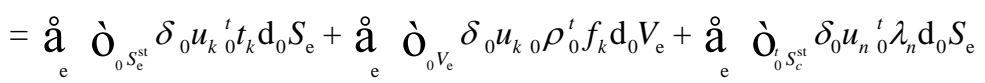

$$
\begin{aligned}
& +\underset{\mathrm{e}}{\stackrel{\circ}{\mathrm{O}}} \grave{\mathrm{O}}_{0} S_{c}^{\mathrm{st}} \delta_{0} \lambda_{n}\left({ }_{0}^{t} u_{n}+{ }_{0}^{0} g_{n}\right) \mathrm{d}_{0} S_{\mathrm{e}}
\end{aligned}
$$

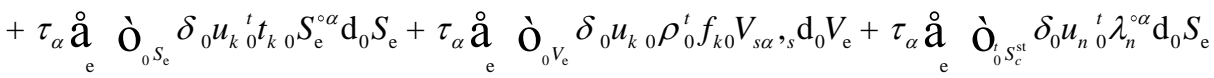

$$
\begin{aligned}
& +\tau_{\alpha} \underset{\mathrm{e}}{\stackrel{\mathrm{a}}{\mathrm{O}_{0}}{ }_{0} S_{c}^{\mathrm{st}}} \delta_{0} \lambda_{n}{ }_{0}^{t} u_{n}^{\circ \alpha} \mathrm{d}_{0} S_{\mathrm{e}}+\tau_{\alpha} \underset{\mathrm{e}}{\stackrel{\circ}{\mathrm{O}}} \grave{\mathrm{O}}_{{ }_{0}} S_{c}^{\mathrm{st}} \delta_{0} u_{n}{ }_{0}^{t} \lambda_{n 0} S_{\mathrm{e}}^{\circ \alpha} \mathrm{d}_{0} S_{\mathrm{e}} \\
& +\tau_{\alpha} \stackrel{\circ}{\mathrm{a}} \grave{\mathrm{O}}_{{ }_{0}} S_{c}^{\mathrm{st}} \delta_{0} \lambda_{n}\left({ }_{0}^{t} u_{n}+{ }_{0}^{0} g_{n}\right)_{0} S_{\mathrm{e}}^{\circ \alpha} \mathrm{d}_{0} S_{\mathrm{e}}+\tau_{\alpha} \tau_{\beta} \stackrel{\mathrm{a}}{\mathrm{e}} \grave{\mathrm{O}}_{{ }_{0} S_{c}^{S_{c}}} \delta_{0} u_{n 0}^{t} \lambda_{n}^{\circ \alpha}{ }_{0}^{\circ} S_{\mathrm{e}}^{\circ \beta} \mathrm{d}_{0} S_{\mathrm{e}} \\
& +\underset{\mathrm{e}}{\mathrm{a}} \tau_{\alpha} \tau_{\beta} \grave{\mathrm{O}}_{0}{ }_{0} \mathrm{Ssc}_{c}^{\mathrm{st}} \delta_{0} \lambda_{n}{ }_{0}^{t} u_{n}^{\circ \alpha}{ }_{0}^{\circ{ }^{\circ \beta}} \mathrm{d}_{0} S_{\mathrm{e}}
\end{aligned}
$$

Neglecting small higher order terms, the terms related to the stress field ${ }_{0}^{t} S_{i j}$ and the loads ${ }_{0}^{t} t_{k},{ }_{0} \rho_{0}^{t} f_{k}$ which are in equilibrium in the original shape, Eq. (A4.4) can be rewritten as 


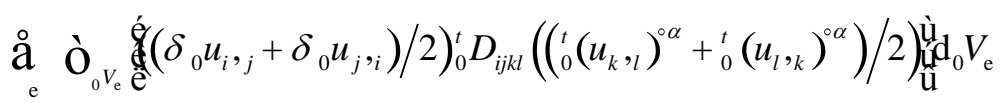

$$
\begin{aligned}
& \left.+\underset{\mathrm{e}}{\mathrm{\textrm {O }}} \mathrm{O}_{0}{ }_{V_{\mathrm{e}}} \stackrel{\dot{d}}{\mathrm{e}}\left(\delta_{0} u_{i},{ }_{j}+\delta_{0} u_{j},,_{i}\right) / 2\right)_{0}^{t} D_{i j k l}\left(\left({ }_{0}^{t} u_{m},{ }_{k}{ }_{0}^{t}\left(u_{m},{ }_{l}\right)^{\circ \alpha}+{ }_{0}^{t} u_{m},{ }_{l}^{t}\left(u_{m},{ }_{k}\right)^{\circ \alpha}\right) / 2\right)_{\mathrm{u}}^{\mathrm{u}} \mathrm{d}_{0} V_{\mathrm{e}}
\end{aligned}
$$

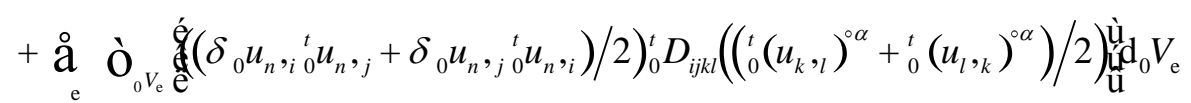

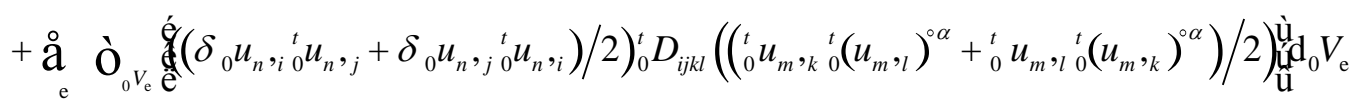

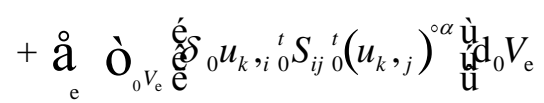

$$
\begin{aligned}
& =\underset{\mathrm{e}}{\stackrel{\mathrm{a}}{\mathrm{O}_{0} S_{\mathrm{e}}^{t}}}\left(\delta_{0} u_{k}\right)\left({ }_{0}^{t} t_{k}\right){ }_{0} S_{\mathrm{e}}^{\circ \alpha} \mathrm{d}_{0} S_{\mathrm{e}}+\underset{\mathrm{e}}{\stackrel{\circ}{\mathrm{O}_{0} V_{\mathrm{e}}}}\left(\delta_{0} u_{k}\right)\left({ }_{0} \rho_{0}^{t} f_{k}\right)_{0} V_{i \alpha},_{i} \mathrm{~d}_{0} V_{\mathrm{e}}
\end{aligned}
$$

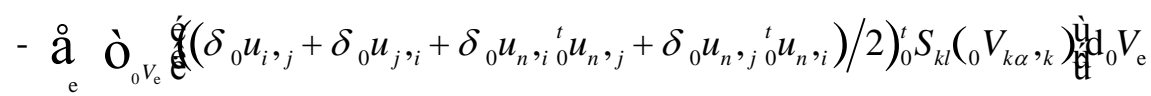

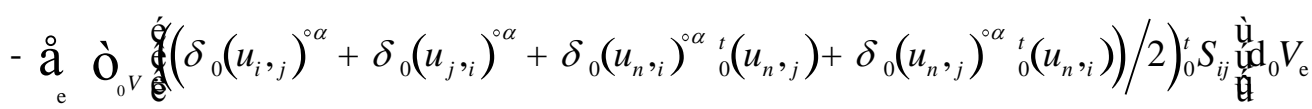

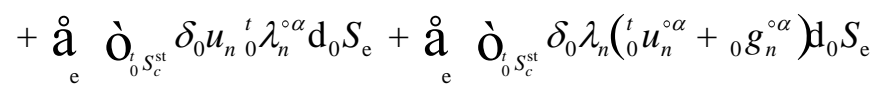

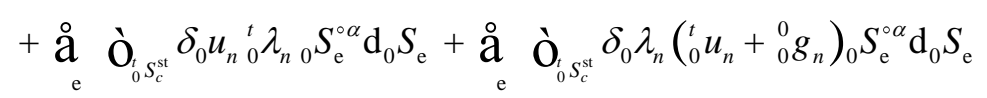

When the structure is modelled using HEX8 element, one can obtain the following weak form control equations

$$
\begin{aligned}
& N^{m}=\frac{1}{8}\left(1+\xi_{1^{\prime}}^{m} \xi_{1^{\prime}}\right)\left(1+\xi_{2^{\prime}}^{m} \xi_{2^{\prime}}\right)\left(1+\xi_{3^{\prime}}^{m} \xi_{3^{\prime}}\right) \\
& { }_{0} x_{j}(\xi)=\sum_{m=1}^{8} N^{m}\left(\xi_{1^{\prime}}, \xi_{2^{\prime}}, \xi_{3^{\prime}}\right)_{0} x_{j}^{m} \\
& { }_{0}^{t} u_{j}(\boldsymbol{\xi})=\sum_{m=1}^{8} N^{m}\left(\xi_{1^{\prime}}, \xi_{2^{\prime}}, \xi_{3^{\prime}}\right){ }_{0}^{t} u_{j}^{m} \\
& \left.{ }_{0}^{t}\left(u_{i},{ }_{j}\right)^{\mathrm{o} \alpha} @ \frac{\mathrm{T}}{\mathrm{T} \tau_{\alpha}}\left({ }_{\tau}^{t} u_{i},{ }_{j}\right)\right|_{\tau=0}={ }_{0}^{t} u_{i}^{\mathrm{o} \alpha},{ }_{j}-{ }_{0}^{t} u_{i},{ }_{k 0} V_{k \alpha},{ }_{j}
\end{aligned}
$$

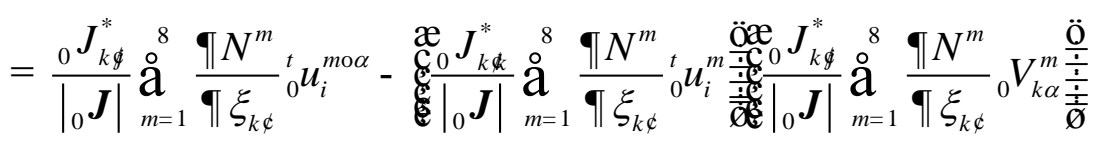




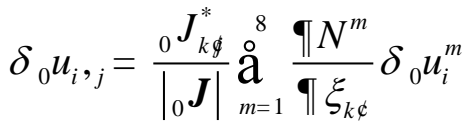

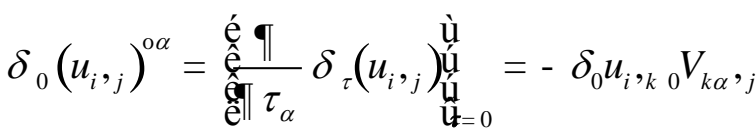

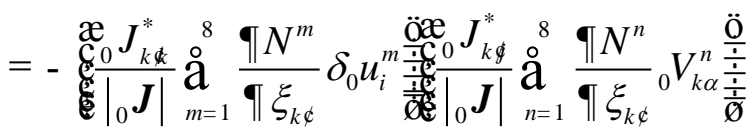

Where

$$
{ }_{0} \boldsymbol{J}\left(\xi_{1^{\prime}}, \quad \xi_{2^{\prime}}, \quad \xi_{3^{\prime}}\right)=\left[\begin{array}{lll}
\frac{\partial_{0} x_{1}}{\partial \xi_{1^{\prime}}} & \frac{\partial_{0} x_{2}}{\partial \xi_{1^{\prime}}} & \frac{\partial_{0} x_{3}}{\partial \xi_{1^{\prime}}} \\
\frac{\partial_{0} x_{1}}{\partial \xi_{2^{\prime}}} & \frac{\partial_{0} x_{2}}{\partial \xi_{2^{\prime}}} & \frac{\partial_{0} x_{3}}{\partial \xi_{2^{\prime}}} \\
\frac{\partial_{0} x_{1}}{\partial \xi_{3^{\prime}}} & \frac{\partial_{0} x_{2}}{\partial \xi_{3^{\prime}}} & \frac{\partial_{0} x_{3}}{\partial \xi_{3^{\prime}}}
\end{array}\right]=\left[\begin{array}{llll}
{ }_{0} J_{11^{\prime}} & { }_{0} J_{21^{\prime}} & { }_{0} J_{31^{\prime}} \\
{ }_{0} J_{12^{\prime}} & { }_{0} J_{22^{\prime}} & { }_{0} J_{32^{\prime}} \\
{ }_{0} J_{13^{\prime}} & { }_{0} J_{23^{\prime}} & { }_{0} J_{33^{\prime}}
\end{array}\right]
$$

Using index symbol, it can be expressed compactly as

$$
{ }_{0} J_{j^{\prime} i}=\sum_{m=1}^{8} \frac{\xi_{j^{\prime} 0}^{m} x_{i}^{m}}{8} \prod_{\substack{n^{\prime}=1 \\ n^{\prime} \neq j^{\prime}}}^{3}\left(1+\xi_{n^{\prime}}^{m} \xi_{n^{\prime}}\right)
$$

where indices $i, j \notin n \not, \cdots$, except those related to $\stackrel{\circ}{\mathrm{a}}$ and $\tilde{\mathrm{O}}$, obey the rules of index symbol including the summation convention, $e_{i \notin \notin k}$ is the permutation symbol. The determinant of the Jacobian matrix can be expressed compactly as

$$
\begin{aligned}
& \left|{ }_{0} \boldsymbol{J}\right|=e_{i^{\prime} k^{\prime}} J_{i^{\prime} 1} J_{j^{\prime} 2} J_{k^{\prime} 3} \\
& =e_{i^{\prime} j^{\prime} k^{\prime}}\left(\sum_{m=1}^{8} \frac{\xi_{i^{\prime} 0}^{m} x_{1}^{m}}{8} \prod_{\substack{n^{\prime}=1 \\
n^{\prime} \neq i^{\prime}}}^{3}\left(1+\xi_{n^{\prime}}^{m} \xi_{n^{\prime}}\right)\left(\sum_{p=1}^{8} \frac{\xi_{j^{\prime} 0}^{p} x_{2}^{p}}{8} \prod_{\substack{s^{\prime}=1 \\
s^{\prime} \neq j^{\prime}}}^{3}\left(1+\xi_{s^{\prime}}^{p} \xi_{s^{\prime}}\right)\right)\left(\sum_{q=1}^{8} \frac{\xi_{k^{\prime} 0}^{q} x_{3}^{q}}{8} \prod_{\substack{t^{\prime}=1 \\
t^{\prime} \neq k^{\prime}}}^{3}\left(1+\xi_{t^{\prime}}^{q} \xi_{t^{\prime}}\right)\right)\right.
\end{aligned}
$$

For the shape variation, the geometrical coordinates for the initial configuration corresponding to the shape variation parameter $\tau_{\alpha}$ can be formulated as

$$
\begin{aligned}
& { }_{\tau}^{0} x_{i}={ }_{0} x_{i}+{ }_{0} V_{i \alpha} \tau_{\alpha}
\end{aligned}
$$

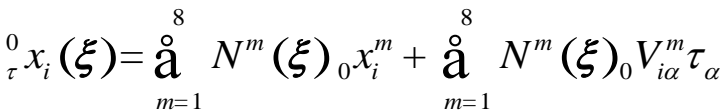


The change rate of the geometrical coordinates of the mapping points with respect to the shape variation parameter $\tau_{a}$ for a generic point and nodal points of an element can be formulated as

$$
{ }_{0} x_{i}^{\circ \alpha}(\xi)=\stackrel{\circ}{\mathrm{a}}_{m=1}^{8} N^{m}(\xi)_{0} V_{i \alpha}^{m} \quad{ }_{0} x_{i}^{m \circ \alpha}={ }_{0} V_{i \alpha}^{m}
$$

Considering Eqs. (A4.6) through (A4.15), the virtual work equation (4.18) and its finite element form can further be rewritten as

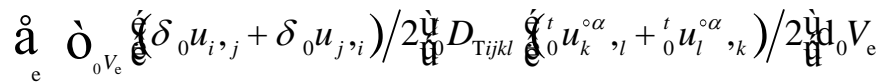

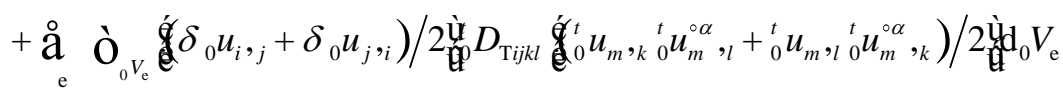

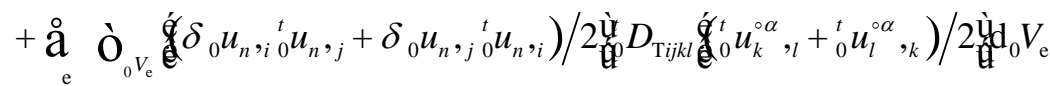

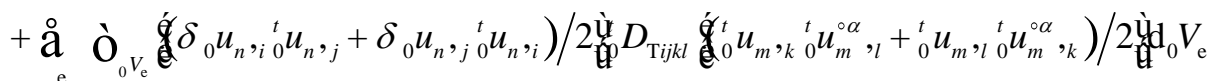

$+\stackrel{\circ}{\mathrm{a}} \mathrm{O}_{0}\left(\delta_{\mathrm{e}}\left(\delta_{0} u_{n},{ }_{i}\right){ }_{0}^{t} S_{i j}\left({ }_{0}^{t} u_{n}^{\circ \alpha},{ }_{j}\right) \mathrm{d}_{0} V_{\mathrm{e}}\right.$

$=\underset{\mathrm{e}}{\stackrel{\circ}{\mathrm{O}}{ }_{0} S_{\mathrm{e}}}\left(\delta_{0} u_{k}\right)\left({ }_{0}^{t} t_{k}\right){ }_{0} S_{\mathrm{e}}^{\circ \alpha} \mathrm{d}_{0} S_{\mathrm{e}}+\underset{\mathrm{e}}{\stackrel{\circ}{\mathrm{O}_{0} V_{\mathrm{e}}}}\left(\delta_{0} u_{k}\right)\left({ }_{0} \rho_{0}^{t} f_{k}\right)_{0} V_{i \alpha}{ }_{i} \mathrm{~d}_{0} V_{\mathrm{e}}$

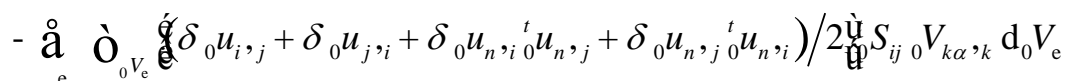

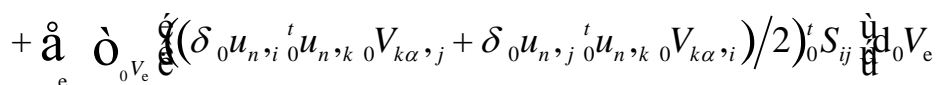

$\left.+\stackrel{\mathrm{a}}{\mathrm{O}} \mathrm{O}_{0} V_{\mathrm{e}} \stackrel{\dot{q}}{c}\left(\delta_{0} u_{i},{ }_{k}^{t} V_{k \alpha},{ }_{j}+\delta_{0} u_{j},{ }_{k}^{t} V_{k \alpha},_{i}\right) / 2\right)_{0}^{t} S_{i j} \grave{\mathrm{d}}_{\mathrm{d}} V_{\mathrm{e}}$

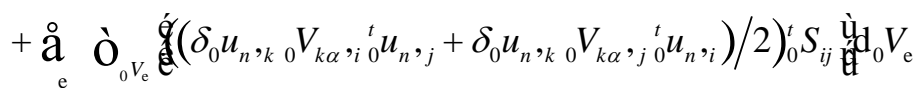

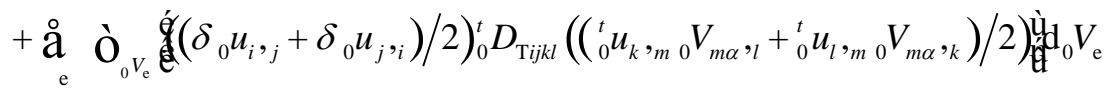

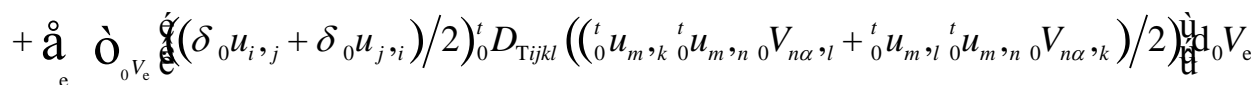

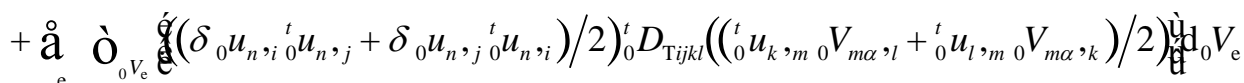

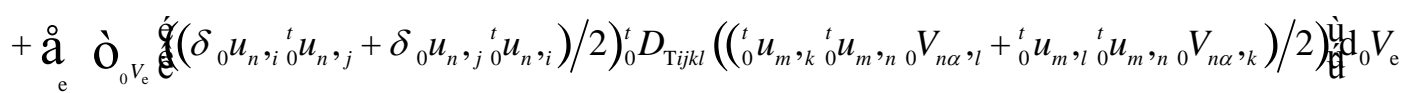

$+\underset{\mathrm{e}}{\stackrel{\circ}{\mathrm{O}_{0}}} \grave{ }_{S_{c}^{\mathrm{st}}}\left(\delta_{0} u_{n}{ }_{0}^{t} \lambda_{n}^{\circ \alpha}+\delta_{0} \lambda_{n}{ }_{0}^{0} g_{n}^{\circ \alpha}+\delta_{0} \lambda_{n}{ }_{0}^{t} u_{n}^{\circ \alpha}+\left(\delta_{0} u_{n}{ }_{0}^{t} \lambda_{n}\right)_{0} S_{\mathrm{e}}^{\circ \alpha}\right) \mathrm{d}_{0} S_{\mathrm{e}}$

Finally, sensitivity equations for state variables can be put in the matrix form of Eq. (24). 